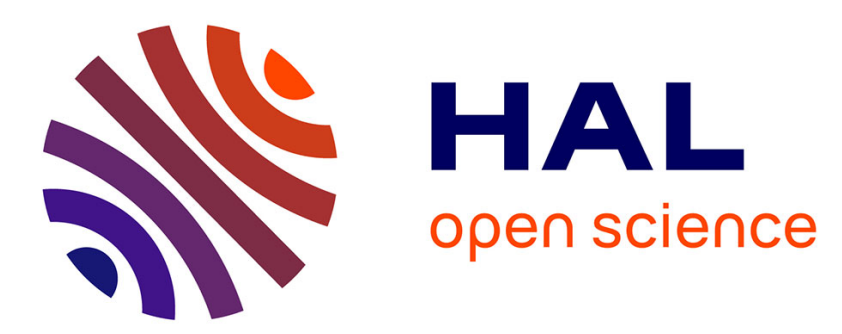

\title{
A Lagrange multiplier method for a discrete fracture model for flow in porous media
}

\author{
Markus Köppel, Vincent Martin, Jérôme Jaffré, Jean E Roberts
}

\section{To cite this version:}

Markus Köppel, Vincent Martin, Jérôme Jaffré, Jean E Roberts. A Lagrange multiplier method for a discrete fracture model for flow in porous media. Computational Geosciences, 2018, 10.1007/s10596018-9779-8 . hal-01700663v3

\section{HAL Id: hal-01700663 \\ https://hal.science/hal-01700663v3}

Submitted on 3 Jan 2019

HAL is a multi-disciplinary open access archive for the deposit and dissemination of scientific research documents, whether they are published or not. The documents may come from teaching and research institutions in France or abroad, or from public or private research centers.
L'archive ouverte pluridisciplinaire HAL, est destinée au dépôt et à la diffusion de documents scientifiques de niveau recherche, publiés ou non, émanant des établissements d'enseignement et de recherche français ou étrangers, des laboratoires publics ou privés. 
Markus Köppel · Vincent Martin · Jérôme Jaffré · Jean E. Roberts

\section{A Lagrange multiplier method for a discrete fracture model for flow in porous media}

February 2018

M. Köppel

Universtität Stuttgart, Institut für Angewandte Analysis und Numerische Simulation (IANS), Pfaffenwaldring 57, 70569

Stuttgart, Germany, E-mail: markus.koeppel@ians.uni-stuttgart.de

V. Martin

Université de Technologie de Compiègne (UTC), Laboratoire de Mathématiques Appliquées de Compiègne (LMAC), Rue du docteur Schweitzer CS 60319, 60203 Compiègne Cedex France, E-mail: vincent.martin@utc.fr

J. Jaffré, J. E. Roberts

Inria Paris, 2 rue Simone Iff, 75589 Paris \& Université Paris-Est, CERMICS (ENPC), 77455 Marne-la-Vallée 2, France,

E-mail: jerome.jaffre@inria.fr

E-mail: jean-elizabeth.roberts@inria.fr

\begin{abstract}
In this work we present a novel discrete fracture model for single-phase Darcy flow in porous media with fractures of co-dimension one, which introduces an additional unknown at the fracture interface. Inspired by the fictitious domain method this Lagrange multiplier couples fracture and matrix domain and represents a local exchange of the fluid. The multipliers naturally impose the equality of the pressures at the fracture interface. The model is thus appropriate for domains with fractures of permeability higher than that in the surrounding bulk domain. In particular the novel approach allows for independent, regular meshing of fracture and matrix domain and therefore avoids the generation of small elements. We show existence and uniqueness of the weak solution of the continuous primal formulation. Moreover we discuss the discrete inf-sup condition of two different finite element formulations. Several numerical examples verify the accuracy and convergence of proposed method.
\end{abstract}

Keywords discrete fracture model · porous media . finite element method - Lagrange multiplier method . nonconforming grids

\section{Introduction}

The subsurface of the earth generally contains a variety of heterogeneous features such as different geological formations, inclusions and fractures. The material parameters in the domain of interest thus may vary by several orders of magnitude. This often leads to a significant change in the flow behavior, in particular if large fractures are present. A fracture is characterized by its lateral dimension which is considerably smaller than its extension in other directions. Depending on their hydrogeological properties, fractures may act as barriers and/or conduits to the flow. Common examples of domains of application in the Earth sciences include $\mathrm{CO}_{2}$ sequestration below caprock formations, underground storage of radioactive waste, geothermal energy production and enhanced oil recovery. In the last few decades, the inclusion of fractures in models for flow in porous media has received more and more attention, and a variety of different models have been proposed.

In principle, fractured porous media models can be categorized roughly as either discrete fracture-matrix (DFM) models or continuum fracture models. Since the proposed method requires information concerning the location of the fractures in the domain of interest and since the method calculates the flow in the fracture as well as in the surrounding domain, we will focus mainly on DFM models in the remainder of this article. We refer to e.g. $[11,50]$ for a more general overview of flow models for fractured porous media. Because of their 
aforementioned geometries, a common way to incorporate fractures in a DFM model is to consider them as $(n-1)$-dimensional objects within the surrounding $n$-dimensional matrix (bulk) domain. This approach avoids the generation of small elements of the spatial discretization grid in (the vicinity of) the fracture and reduces the computational costs. Additionally, it is often assumed that the fracture is filled with debris which facilitates the modeling by making it appropriate to use Darcy's law in both the fracture and matrix parts of the domain. Such models have been extensively studied from the mathematical and/or the engineering point of view. Many of these studies are concerned with linear Darcy flow; see $[5,6,7,10,16,22,25,26,46,58]$, to name just a few. Others represent extensions to allow for Forchheimer flow in the fractures, [29,41], or for DarcyBrinkman flow, [44], and others for two-phase or multiphase flow, $[1,17,18,30,33,35,36,38,39,45,47,48,49]$, where again we cite just a few. Some articles have also taken up the topic of discrete fracture network (DFN) models, e.g. $[12,13,51]$. Various numerical discretization methods have been used: finite element methods $[10,40]$, mixed or mixed-hybrid finite elements, $[5,6,46]$, finite volume methods, [7,25,35,39,52,54], multi-point flux methods, [2,3,54], mimetic finite difference methods, [9], discontinuous Galerkin methods, [8], vertex approximate -gradient methods, $[16,17]$. Still another approach was given in [15].

In many articles the fracture elements, of codimension one with respect to the matrix domain, coincide with the faces of the matrix elements. This configuration is generally referred to as a matching fracture and matrix grid approach. However one may wish to discretize the fracture more finely in the case of a highly conductive fracture or more coarsely in the case of a barrier and methods allowing for non-matching grids may be used; see e.g. $[26,28,58]$. Still with these methods the fracture can not cut through the interior of a matrix element; it must lie in the union of the faces of the matrix elements. The matrix grid must be aligned with the fracture.

Nonconforming methods, on the other hand, are characterized by an independent meshing of the fracture and the matrix domain which allows for regular meshes and elements in the corresponding domains. The most prominent example in the field of nonconforming methods is the extended finite element method (XFEM), e.g. in $[22,37,55]$ for the primal formulation and in $[23$, 31] for the dual formulation, where the respective basis functions are locally enriched in the vicinity of the fracture to account for the discontinuities.

This paper presents an alternative nonconforming formulation. The method uses Lagrange multiplier variables in a primal variational formulation to connect the fracture flow with the flow in the matrix. The multipliers approximate the jump of the normal flux across the fracture interface and represent the exchange between the fracture and the matrix. Using the ideas of [34] we show that the continuous problem is well posed. In this paper the pressure is assumed to be continuous across the fracture, i.e. the permeability in the fracture is assumed to be larger than in the matrix. The case of geological barriers is thus excluded from the current study. The discretization uses Lagrange $\mathbb{P}^{1}$ finite elements both in the matrix and in the fracture, and since the exchanges between the fracture and matrix flow are only through Lagrange multipliers, the grids for the matrix and the fracture can be mutually independent. The multipliers are discretized by either piecewise constant or continuous, piecewise linear basis functions on the fracture interface provided that the involved mesh size is not too small compared to the matrix mesh. We show the inf-sup stability of the first of these, again following ideas of [34]. Somewhat surprisingly, the fracture flow equation does not figure in the proof of the stability and the pressure fracture mesh can be chosen arbitrarily. In a companion paper [43], we study a different discretization with a consistent penalty term to stabilize the system and a different way of treating the mesh compatibility issue.

The remainder of the paper is organized as follows: in Section 2, we give the continuous formulation of the Lagrange-multiplier, finite element method and prove the existence and uniqueness of the weak solution for a domain of dimension 2 or 3 . Section 3 concerns the discrete formulations of the problem and the proof of their well-posedness. For this part we have considered only the case of a 2 dimensional domain. In Section 4, we analyze the method by means of several numerical examples of different complexity. We perform a numerical error and convergence analysis to study the constraints on the mesh size of the multipliers and the performance of the method in more detail. Finally we conclude and discuss the proposed method in Section 5.

\section{A Lagrange multiplier formulation of the continuous problem}

We consider a convex, matrix domain $\Omega \subset \mathbb{R}^{n}, n=2$ or 3 , and a fracture domain $\gamma \subset \Omega$ of dimension $n-1$, with a continuous unit vector field $\mathbf{n}_{\gamma}$ normal to the fracture-surface $\gamma$, see Fig. 1. For simplicity we assume that the fracture $\gamma$ is a line segment if $n=2$ and a planar surface if $n=3$, and that $\partial \gamma \subset \partial \Omega$. Also for simplicity homogeneous Dirichlet boundary conditions on $\partial \Omega$ and on $\partial \gamma$ are imposed. Flow in $\Omega$ is governed by

$$
\begin{array}{ll}
\operatorname{div}(-\mathbf{K} \nabla p)=f & \text { in } \Omega \\
p=0 & \text { on } \Gamma=\partial \Omega
\end{array}
$$

and in $\gamma$ by

$$
\begin{array}{ll}
\operatorname{div}_{\gamma}\left(-\mathbf{K}_{\gamma} \nabla_{\gamma} p_{\gamma}\right)=f_{\gamma} & \text { in } \gamma \\
p_{\gamma}=0 & \text { on } \partial \gamma,
\end{array}
$$

where $\operatorname{div}_{\gamma}$ and $\nabla_{\gamma}$ are the $(n-1)$-dimensional divergence and gradient operators in the plane of $\gamma$, the 


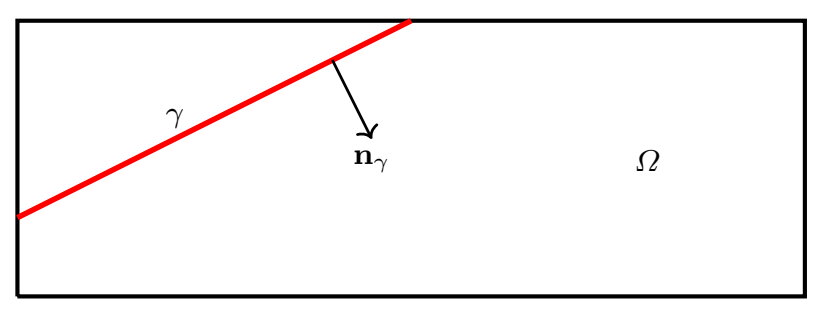

Fig. 1 Example of a domain containing a fracture.

coefficients $\mathbf{K}$ and $\mathbf{K}_{\gamma}$ are the symmetric, uniformly positive-definite, bounded, permeability tensor-fields on $\Omega$ and $\gamma$ respectively, the unknowns $p$ and $p_{\gamma}$ represent the fluid pressure and $f$ and $f_{\gamma}$ external source terms. However, to permit the possibility of fluid exchange between $\Omega$ and $\gamma$, we introduce a term $\lambda=\lambda(x), x \in \gamma$, that will be added in as a source/sink term in $\gamma$ and subtracted out as a sink/source term in $\Omega$ at its intersection with $\gamma$. Thus equations (1) and (2) become

$$
\begin{array}{ll}
\operatorname{div}(-\mathbf{K} \nabla p)-\lambda=f, & \text { in } \Omega, \\
p=0, & \text { on } \Gamma=\partial \Omega,
\end{array}
$$

and

$$
\begin{array}{ll}
\operatorname{div}_{\gamma}\left(-\mathbf{K}_{\gamma} \boldsymbol{\nabla}_{\gamma} p_{\gamma}\right)+\lambda=f_{\gamma}, & \text { in } \gamma, \\
p_{\gamma}=0, & \text { on } \partial \gamma
\end{array}
$$

To obtain a variational formulation we will multiply by test functions $q$ and $q_{\gamma}$, integrate over $\Omega$ and $\gamma$, and use integration by parts in both equations. Define the spaces $V_{\Omega}, V_{\gamma}, \boldsymbol{V}$ and $\Lambda$ as follows:

$$
\begin{aligned}
V_{\Omega}=H_{0}^{1}(\Omega), & V_{\gamma}=H_{0}^{1}(\gamma) \\
\boldsymbol{V}=V_{\Omega} \times V_{\gamma}, & \Lambda=H_{0,0}^{-\frac{1}{2}}(\gamma) .
\end{aligned}
$$

These spaces are endowed with the following norms: for $q \in V_{\Omega}$, for $q_{\gamma} \in V_{\gamma}$ and for $\mu \in \Lambda$,

$$
\begin{aligned}
& \|q\|_{V_{\Omega}}^{2}=\|q\|_{0, \Omega}^{2}+\|\nabla q\|_{0, \Omega}^{2}, \\
& \left\|q_{\gamma}\right\|_{V_{\gamma}}^{2}=\left\|q_{\gamma}\right\|_{0, \gamma}^{2}+\left\|\nabla_{\gamma} q_{\gamma}\right\|_{0, \gamma}^{2}, \\
& \left\|\left(q, q_{\gamma}\right)\right\|_{\boldsymbol{V}}^{2}=\|q\|_{V_{\Omega}}^{2}+\left\|q_{\gamma}\right\|_{V_{\gamma}}^{2} \text {, } \\
& \|\mu\|_{\Lambda}=\sup _{\xi \in H_{0,0}^{\frac{1}{2}}(\gamma)} \frac{\int_{\gamma} \mu, \xi}{\|\xi\|_{H_{0,0}^{\frac{1}{2}}(\gamma)}},
\end{aligned}
$$

where $\|\cdot\|_{0, \mathcal{O}}$ denotes the standard $L^{2}(\mathcal{O})$ norm on an open set $\mathcal{O}$, where $\|\cdot\|_{H_{0,0}^{\frac{1}{2}}(\gamma)}$ is defined by $\|\xi\|_{H_{0,0}^{\frac{1}{2}}(\gamma)}=$ $\inf _{q \in V_{\Omega}}\|q\|_{V_{\Omega}}$, where for $q \in V_{\Omega},\left.q\right|_{\gamma}$ denotes the $\left.q\right|_{\gamma}=\xi$

trace of $q$ on $\gamma$ and where $\int_{\gamma} \mu \xi$ denotes the duality pairing $\langle\cdot, \cdot\rangle_{\gamma}$ between $H_{0,0}^{-\frac{1}{2}}(\gamma)$ and $H_{0,0}^{\frac{1}{2}}(\gamma)$. The variational formulation is then given as follows:

Find $\left(p, p_{\gamma}\right) \in \boldsymbol{V}$ and $\lambda \in \Lambda$ such that

$$
\begin{array}{ll}
\int_{\Omega} \mathbf{K} \nabla p \cdot \nabla q+\int_{\gamma} \mathbf{K}_{\gamma} \nabla_{\gamma} p_{\gamma} \cdot \nabla_{\gamma} q_{\gamma} & \\
\quad-\int_{\gamma} \lambda\left(\left.q\right|_{\gamma}-q_{\gamma}\right)=\int_{\Omega} f q+\int_{\gamma} f_{\gamma} q_{\gamma} & \forall\left(q, q_{\gamma}\right) \in \boldsymbol{V} \\
\int_{\gamma}\left(\left.p\right|_{\gamma}-p_{\gamma}\right) \mu=0 & \forall \mu \in \Lambda,
\end{array}
$$

where the Lagrange multiplier can be interpreted as the jump across the fracture of the normal component of the product of the permeability tensor with the gradient of the matrix pressure, i.e. $\lambda=\llbracket \mathbf{K} \nabla p \cdot \mathbf{n}_{\gamma} \rrbracket_{\gamma}$.

Problem (7) is a Lagrange multiplier primal formulation that corresponds to the fracture problem that was studied in $[5,6]$ in mixed form. Then defining the bilinear forms $a: \boldsymbol{V} \times \boldsymbol{V} \longrightarrow \mathbb{R}$ and $b: \boldsymbol{V} \times \Lambda \longrightarrow \mathbb{R}$ and the linear form $\ell: \boldsymbol{V} \longrightarrow \mathbb{R}$ by

$a\left(\left(p, p_{\gamma}\right),\left(q, q_{\gamma}\right)\right)=\int_{\Omega} \mathbf{K} \boldsymbol{\nabla} p \cdot \nabla q+\int_{\gamma} \mathbf{K}_{\gamma} \nabla_{\gamma} p_{\gamma} \cdot \nabla_{\gamma} q_{\gamma}$,

$b\left(p, p_{\gamma} ; \mu\right)=\int_{\gamma}\left(\left.p\right|_{\gamma}-p_{\gamma}\right) \mu$,

$\ell\left(q, q_{\gamma}\right)=\int_{\Omega} f q+\int_{\gamma} f_{\gamma} q_{\gamma}$,

we may write (7) in standard mixed form,

Find $\left(p, p_{\gamma}\right) \in \boldsymbol{V}$ and $\lambda \in \Lambda$ such that

$$
\begin{array}{lc}
a\left(\left(p, p_{\gamma}\right),\left(q, q_{\gamma}\right)\right)-b\left(q, q_{\gamma} ; \lambda\right)=\ell\left(q, q_{\gamma}\right), & \forall\left(q, q_{\gamma}\right) \in \boldsymbol{V} \\
b\left(p, p_{\gamma} ; \mu\right)=0, & \forall \mu \in \Lambda,
\end{array}
$$

and, following the techniques of [34], prove the following theorem:

Theorem 1 There exists a unique solution to the continuous Lagrange multiplier, fracture problem (9).

Proof It is easily seen that the bilinear forms $a$ and $b$ are continuous and that, in light of the hypotheses made concerning $\mathbf{K}$ and $\mathbf{K}_{\gamma}, a$ is elliptic on all of $\boldsymbol{V}$. Thus in order to show the existence and uniqueness of the solution of (9) using the classical results of Brezzi $[14,20,53]$ we have only to show that $b(\cdot, \cdot)$ satisfies an inf-sup condition; more precisely, to show that there exists a positive constant $\theta_{b}$ such that

$$
\inf _{\mu \in \Lambda} \sup _{\left(q, q_{\gamma}\right) \in \boldsymbol{V}} \frac{b\left(q, q_{\gamma} ; \mu\right)}{\|\mu\|_{\Lambda}\left\|\left(q, q_{\gamma}\right)\right\|_{\boldsymbol{V}}} \geq \theta_{b}
$$

Toward this end, recalling the definitions of the $\Lambda$ norm (6) and of the $H_{0,0}^{\frac{1}{2}}(\gamma)$ norm, we note that for 
each $\mu \in \Lambda$

$$
\begin{aligned}
& \sup _{\left(q, q_{\gamma}\right) \in \boldsymbol{V}} \frac{b\left(q, q_{\gamma} ; \mu\right)}{\|\mu\|_{\Lambda}\left\|\left(q, q_{\gamma}\right)\right\|_{\boldsymbol{V}}} \geq \sup _{(q, 0) \in \boldsymbol{V}} \frac{b(q, 0 ; \mu)}{\|\mu\|_{\Lambda}\|(q, 0)\|_{\boldsymbol{V}}} \\
& =\sup _{q \in V_{\Omega}} \frac{\left\langle\mu,\left.q\right|_{\gamma}\right\rangle_{\gamma}}{\|\mu\|_{\Lambda}\|q\|_{V_{\Omega}}}=\sup _{\operatorname{seH}_{0,0}^{\frac{1}{2}}(\gamma)} \sup _{\substack{\left.q \in V_{\Omega} \\
q\right|_{\gamma}=\xi}} \frac{\langle\mu, \xi\rangle_{\gamma}}{\|\mu\|_{\Lambda}\|q\|_{V_{\Omega}}} \\
& =\sup _{\xi \in H_{0,0}^{\frac{1}{2}}(\gamma)}\left(\frac{\langle\mu, \xi\rangle_{\gamma}}{\|\mu\|_{\Lambda}} \frac{1}{\inf _{\substack{\left.q \in V_{\Omega} \\
q\right|_{\gamma}=\xi}}\|q\|_{V_{\Omega}}}\right) \\
& =\sup _{\xi \in H_{0,0}^{\frac{1}{2}}(\gamma)} \frac{\langle\mu, \xi\rangle_{\gamma}}{\|\mu\|_{\Lambda}\|\xi\|_{H_{0,0}^{\frac{1}{2}}(\gamma)}}=1 .
\end{aligned}
$$

\section{Discretization}

In this section we are concerned with formulating and analyzing a discrete counterpart to (9). The discrete formulation is based on three distinct meshes, one for the approximation of the pressure in the domain $\Omega$, one for the approximation of the pressure in the fracture $\gamma$, and one for the approximation of the Lagrange multiplier in the fracture. We will in fact consider two discrete problems which will differ in the choice of the space of Lagrange multipliers. In one the multipliers are piecewise constant and in the other they are continuous, piecewise linear functions on $\gamma$. For the case of piecewise constant multipliers, existence and uniqueness as well as convergence are proved, following the ideas of [34], under the hypothesis that the Lagrange multiplier space is not too rich with respect to the space for the approximation of the matrix pressure; i.e. when the Lagrange multiplier mesh size $h_{\lambda}$ is not too small with respect to the matrix pressure mesh size $h$; see Hypothesis 1 and Remark 1. The numerical experiments of Section 4 give accurate results when $h_{\lambda} \geq 2 h$. For the case of continuous multipliers, existence and uniqueness is shown for the case that the mesh, and indeed the approximation space, for the Lagrange multipliers is the same as that for the fracture pressure. We were not able to show convergence in this case, however the numerical results are more than encouraging, see Section 4.

For the remainder of this article we restrict our attention to the two-dimensional setting, i.e. we suppose that $\Omega$ is two-dimensional and $\gamma$ is one-dimensional. We see no inherent reason why the same procedure could not be used in a three-dimensional setting though it would of course be more technically complex and much more involved to implement numerically.

\subsection{Approximation spaces and a discrete formulation}

To discretize problem (9) we let $\mathcal{T}_{h}$ be a finite element discretization of $\Omega$ made up of triangles and/or rectangles, and let both $\mathcal{T}_{h, \gamma}$ and $\mathcal{T}_{h, \lambda}$ be discretizations of $\gamma$ (made up of line segments), and we let $h, h_{\gamma}$, and $h_{\lambda}$ denote the respective mesh sizes:

$$
\begin{aligned}
& h=\max _{T \in \mathcal{T}_{h}} h_{T} \text { where } h_{T}=\operatorname{diam} T, \\
& h_{\gamma}=\max _{t \in \mathcal{T}_{h, \gamma}} h_{t} \text { where } h_{t}=\operatorname{diam} t, \\
& h_{\lambda}=\max _{s \in \mathcal{T}_{h, \lambda}} h_{s} \quad \text { where } h_{s}=\operatorname{diam} s .
\end{aligned}
$$

We assume that each of these grids belongs to a family of discretizations that is regular, and $\mathcal{T}_{h}$ to a family that is uniformly regular in the sense that there is a positive constant $\sigma_{\Omega}$ such that for each $h$,

$$
\frac{h}{\rho_{h}} \leq \sigma_{\Omega}, \text { with } \rho_{h}=\min _{T \in \mathcal{T}_{h}} \rho_{T} \text { and } \rho_{T}=\operatorname{inradius} T \text {. }
$$

We will also make use of the notation $\sigma_{T}=h_{T} / \rho_{T}$.

Let $V_{h, \Omega}$ and $V_{h, \gamma}$ respectively be the space of continuous, piecewise-bilinear (piecewise-affine) functions on $\Omega$ and on $\gamma$ respectively which vanish on $\partial \Omega$ and on $\partial \gamma$ respectively:

$$
\begin{aligned}
& V_{h, \Omega}=\left\{q \in H_{0}^{1}(\Omega): \forall T \in \mathcal{T}_{h},\right. \\
& \left.q\right|_{T} \in\left\{\begin{array}{l}
\mathbb{P}^{1}(T) \text { if } T \text { is a triangle } \\
\mathbb{Q}^{1,1}(T) \text { if } T \text { is a rectangle }
\end{array}\right\}, \\
& V_{h, \gamma}=\left\{q_{\gamma} \in H_{0}^{1}(\gamma): \forall t \in \mathcal{T}_{h, \gamma},\left.\quad q_{\gamma}\right|_{t} \in \mathbb{P}^{1}(t)\right\} .
\end{aligned}
$$

Then $\boldsymbol{V}_{h}$ will denote $V_{h, \Omega} \times V_{h, \gamma}$ :

$$
\boldsymbol{V}_{h}=V_{h, \Omega} \times V_{h, \gamma} .
$$

For the approximation of the Lagrange multiplier $\lambda$, we will consider two different discrete spaces $\Lambda_{h}$ which are defined in Sections 3.2 and 3.3, respectively. Each of these choices for $\Lambda_{h}$ gives rise to a discrete problem whose variational formulation may be expressed as follows:

Find $\left(p_{h}, p_{\gamma, h}\right) \in \boldsymbol{V}_{h}$ and $\lambda_{h} \in \Lambda_{h}$ such that

$$
\begin{aligned}
a\left(\left(p_{h}, p_{\gamma, h}\right),\left(q_{h}, q_{\gamma, h}\right)\right)-b\left(q_{h}, q_{\gamma, h} ; \lambda_{h}\right) & =\ell\left(q_{h}, q_{\gamma, h}\right), \\
& \forall\left(q_{h}, q_{\gamma, h}\right) \in V_{h} \\
b\left(p_{h}, p_{\gamma, h} ; \mu_{h}\right)=0, & \forall \mu_{h} \in \Lambda_{h} .
\end{aligned}
$$

Clearly, on the discrete approximation spaces $\boldsymbol{V}_{h}$, the bilinear form $a$ is both continuous and elliptic and both of these uniformly in $h$.

\subsection{A piecewise constant Lagrange multiplier space}

For the approximation of the Lagrange multiplier $\lambda$ we define the approximation space $\Lambda_{h}$ as follows:

$$
\Lambda_{h}=\left\{\lambda_{h} \in L^{2}(\gamma)\left|\lambda_{h}\right|_{s} \in \mathbb{P}^{0}(s), \forall s \in \mathcal{T}_{h, \lambda}\right\} .
$$

With this definition the bilinear form $b$ is continuous on $\boldsymbol{V}_{h} \times \Lambda_{h}$ with a constant of continuity that is independent of $h$. Thus to apply the standard Brezzi theory for 
mixed formulations and obtain existence and uniqueness of the solution of the discrete problem and to obtain error estimates we need only establish a uniformin- $h$, discrete inf-sup condition. As in the proof of Theorem 1 we actually show the inf-sup condition for $b$ on $\left(V_{\Omega} \times 0\right) \times \Lambda$, we need only define for each $h$, a continuous projection operator $\Pi_{h}: V_{\Omega} \longrightarrow V_{h, \Omega}$ in such a way that the family of projections operators is stable in the sense that the constant of continuity is independent of $h$, and such that $\forall h$ the following diagram commutes:

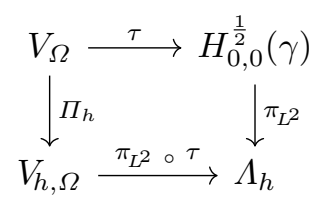

where $\tau: V_{\Omega} \rightarrow H_{0,0}^{\frac{1}{2}}(\gamma)$ denotes the trace map and $\pi_{L^{2}}: L^{2}(\gamma) \rightarrow \Lambda_{h}$ the $L^{2}$-projection operator onto $\Lambda_{h}$.

Following [34], we will define the continuous operators $\Pi_{h}$ such that there exists a constant $C_{\Pi}$ independent of $h$ such that the following two conditions are satisfied:

Condition $1 \forall p \in V_{\Omega}, \quad\left\|\Pi_{h} p\right\|_{1, \Omega} \leq C_{\Pi}\|p\|_{1, \Omega}$,

Condition $2 \forall p \in V_{\Omega}$, and $\forall \mu_{h} \in \Lambda_{h}$,

$$
b\left(p-\Pi_{h} p, 0 ; \mu_{h}\right)=0 .
$$

To define such an operator $\Pi_{h}$ we will use the ScottZhang projection [56], but in order to have Condition 2, we will need to modify this projection operator in the vicinity of $\gamma$, and for this we will need a compatibility condition between $\mathcal{T}_{h}$ and $\mathcal{T}_{h, \lambda}$. The mesh $\mathcal{T}_{h, \gamma}$ does not come into play. We first define some notation associated with the mesh $\mathcal{T}_{h}$. For $T \in \mathcal{T}_{h}$ let $\Delta_{T}$ denote the interior of the union of the closures of the elements $T^{\prime} \in \mathcal{T}_{h}$ sharing a boundary point with $T$, and for a vertex $a$ of some $T \in \mathcal{T}_{h}$, let $\Delta_{a}$ denote the interior of the union of the closures of the elements $T^{\prime} \in \mathcal{T}_{h}$ having $a$ as a vertex, see Fig. 2. For $y$ and $z$ in $\Omega$, let $\overline{y z}$ denote the line segment between $y$ and $z$ and $|y z|$ its length. The condition of compatibility between the meshes $\mathcal{T}_{h}$ and $\mathcal{T}_{h, \lambda}$ needed to define the operators $\Pi_{h}$ is that the following hypothesis holds:

Hypothesis 1 To each $s \in \mathcal{T}_{h, \lambda}$ a vertex $a_{s}$ (of a cell in $\mathcal{T}_{h}$ ) may be associated in such a way that

- there is an edge $e_{s}$ of a cell of $\mathcal{T}_{h}$ and a point $x_{s} \in\left(e_{s} \cap s\right)$ such that $a_{s}$ is an endpoint of $e_{s}$ and $\left|a_{s} x_{s}\right| \leq\left|x_{s} b_{s}\right|$ where $b_{s}$ is the other endpoint of $e_{s}$, - neither endpoint of $s$ belongs to $\Delta_{a_{s}}$

- if $s$ and $s^{\prime}$ are distinct elements of $\mathcal{T}_{h, \lambda}$, then $\Delta_{a_{s}} \cap \Delta_{a_{s^{\prime}}}=\emptyset$.

Note that Hypothesis 1 does not exclude the case in which the fracture $\gamma$ passes through a vertex. (It is possible in some cases to choose for $a_{s}$ a vertex lying on $s$ and to take $x_{s}=a_{s}$ ). Neither does it exclude the case in which $\gamma$ lies along one or more of the edges of the cells of $\mathcal{T}_{h}$. (One can choose the edge $e_{s}$ to be a subset of $s$.)

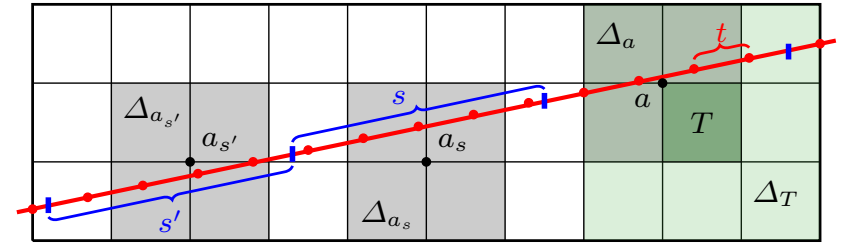

Fig. 2 Construction and support of macro elements. Here all of the elements $T$ are rectangles, but the case of triangular elements is similar. Patches of elements $\Delta_{T}$ are in shades of green and patches of vertices $\Delta_{a_{s}}$ are depicted in grey.

Remark 1 Hypothesis 1 is satisfied if $3 h<|s|, \forall s \in$ $\mathcal{T}_{h, \lambda}$, cf. [34].

For the demonstrations we will also need a second hypothesis:

Hypothesis 2 There exists an $L$ such that $|s| \leq L h$, $\forall s \in \mathcal{T}_{h, \lambda}$.

Remark 2 As the fracture is supposed to be at least as permeable as the matrix domain, normally one would take $h_{\gamma} \leq h$; however, neither the ratio $h / h_{\gamma}$ nor $h_{\lambda} / h_{\gamma}$ is used in the proofs.

Remark 3 The two Conditions 1 and 2 are necessary and sufficient to prove the stability of problem (11). Hypotheses 1 and 2 taken together are sufficient to obtain these two Conditions, see Lemma 3, but are not necessary in general. In particular, the practical requirement on the meshes $3 h<|s|$ (see Remark 1) can often be relaxed, see Section 4.

\subsubsection{A multiplicative trace lemma}

Before establishing approximation results we give a multiplicative trace inequality.

Lemma 1 There is a constant $C_{\tau}>0$ such that if $T$ is a triangle, $\zeta$ is a straight line segment contained in $T$ and $q \in H^{1}(T)$, then

$$
\left\|\left.q\right|_{\gamma}\right\|_{0, \zeta}^{2} \leq C_{\tau} \sigma_{T}\left(h_{T}^{-1}\|q\|_{0, T}^{2}+h_{T}\|\nabla q\|_{0, T}^{2}\right) .
$$

Proof Let $T$ be a triangle and $\zeta$ a line segment with $\zeta \subset T$. We use a bijective affine transformation from a reference element $\widehat{T}$ onto $T$. Let $\hat{\zeta}$ denote the preimage of $\zeta$ under this transformation, and for $q \in H^{1}(T)$ let $\hat{q} \in H^{1}(\widehat{T})$ denote the induced function on $\widehat{T}$. Applying now [34, Lemma 2], which states that there is a constant $\widehat{C}$ such that $\forall \hat{r} \in H^{1}(\widehat{T})$ and for each line segment $\hat{\xi} \subset \widehat{T}$

we obtain

$$
\left\|\left.\hat{r}\right|_{\hat{\xi}}\right\|_{0, \hat{\xi}} \leq \widehat{C}\|\hat{r}\|_{1, \widehat{T}}
$$

$$
\begin{aligned}
\left\|\left.q\right|_{\zeta}\right\|_{0, \zeta}^{2} & \leq\left\|B_{T}\right\|\left\|\left.\hat{q}\right|_{\widehat{\zeta}}\right\|_{0, \widehat{\zeta}}^{2} \\
& \leq \widehat{C}^{2}\left\|B_{T}\right\|\|\hat{q}\|_{1, \widehat{T}}^{2}=\widehat{C}^{2}\left\|B_{T}\right\|\left(\|\hat{q}\|_{0, \widehat{T}}^{2}+|\hat{q}|_{1, \widehat{T}}^{2}\right) \\
& \leq \widehat{C}^{2}\left\|B_{T}\right\|\left|\operatorname{det}\left(\mathrm{B}_{\mathrm{T}}\right)\right|^{-1}\left(\|\mathrm{q}\|_{0, \mathrm{~T}}^{2}+\left\|\mathrm{B}_{\mathrm{T}}\right\|^{2}|\mathrm{q}|_{1, \mathrm{~T}}^{2}\right) \\
& \leq c \widehat{C}^{2} h_{T}\left(h_{T} \rho_{T}\right)^{-1}\left(\|q\|_{0, T}^{2}+h_{T}^{2}|q|_{1, T}^{2}\right),
\end{aligned}
$$


where $B_{T}$ is the linear part of the affine transformation from $\widehat{T}$ to $T$, and $c$ is independent of $h_{T}$.

Remark 4 Lemma 1 also holds if $T$ is instead a rectangle. The proof is completely analagous to the one above.

This lemma cannot be derived directly from [19, Theorem 1.6.6] (together with Young's inequality), because the constant in that Theorem depends on the domain ( $T$ here). It could though be derived from [4], using the fact that a triangle can be chosen inside $T$ as such: it has an edge that is $\zeta$ and the minimum and maximum lengths between $\zeta$ and the opposite vertex are bounded by $h_{T}$ from below and from above. Thus one can apply [4, Lemma 10] using $\zeta$ as the edge of this particular triangle and recover an estimate similar to (13). See also [?, Lemma 9.14] for the case of $L^{p}$.

\subsubsection{Existence, uniqueness and convergence}

To prove the well-posedness of Problem (11), with $\Lambda_{h}$ defined in (12), under Hypotheses 1 and 2, following [34], we define the projection operator to be a locally corrected Scott-Zhang interpolation operator:

$$
\Pi_{h} p=\mathcal{S} Z_{h} p+\sum_{s \in \mathcal{T}_{h, \lambda}} C_{s} \phi_{s}, \quad \text { for } p \in V_{\Omega},
$$

where $\mathcal{S} Z_{h}: V_{\Omega} \longrightarrow V_{h, \Omega}$ is the Scott-Zhang interpolation operator, $\phi_{s} \in V_{h, \Omega}$ is the function having nodal values equal to zero except at $a_{s}$ where the nodal value is 1 , and the constant $C_{s}$ is defined by

$$
C_{s}=\left.\frac{-1}{\left.\int_{s} \phi_{s}\right|_{s}} \int_{s}\left(\mathcal{S} Z_{h} p-p\right)\right|_{s}
$$

The next two lemmas show that the operator $\Pi_{h}$ satisfies Conditions 1 and 2 .

Lemma 2 Assume that the pair of meshes $\left(\mathcal{T}_{h}, \mathcal{T}_{h, \lambda}\right)$ satisfies Hypothesis 1. Then there exists a constant $C_{\phi}>$ 0 independent of $h$ such that for each $s \in \mathcal{T}_{h, \lambda}$

$$
\left.\int_{s} \phi_{s}\right|_{s} \geq C_{\phi} h
$$

Proof We give the proof for the case in which the mesh $\mathcal{T}_{h}$ is a mesh of triangles. The case of rectangles is similar. For $s \in \mathcal{T}_{h, \lambda}$ let $a_{s}, e_{s}, x_{s}$ and $b_{s}$ be as in Hypothesis 1 . Then $e_{s}$ borders two cells lying in $\Delta_{a_{s}}$. Choose one and label it $T_{s}$, and label its remaining vertex $c_{s}$, see Fig. 3, left. The angle of $T_{s}$ at $b_{s}$ will be denoted by $\widehat{b_{s}}$. Denote by $y_{s}$ the endpoint of $T_{s} \cap s$ opposite $x_{s}$ and by $\delta_{s}$ the length of $\overline{x_{s} y_{s}}$, the segment between $x_{s}$ and $y_{s}$. Since $\phi_{s}$ is linear in $T_{s}$, from the definition of $x_{s}$ we have

$$
\left.\int_{T_{s} \cap s} \phi_{s}\right|_{T_{s} \cap s}=\frac{1}{2}\left(\phi_{s}\left(x_{s}\right)+\phi_{s}\left(y_{s}\right)\right) \delta_{s} \geq \frac{1}{4} \delta_{s} .
$$
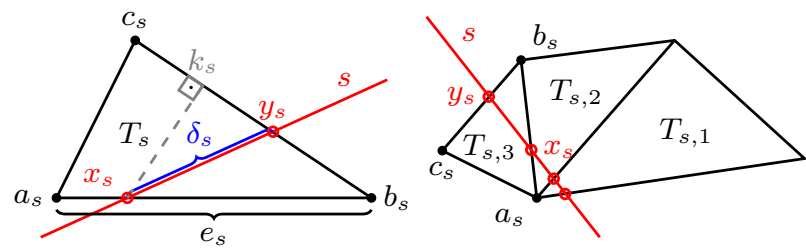

Fig. 3 Intersections of a macro element on a triangular grid.

There remains to find a lower bound for $\delta_{s}$ in terms of $h$. There are three possible cases: in the first case $s \cap e_{s}=e_{s}$. In this case one can take $x_{s}=a_{s}$ and $y_{s}=b_{s}$, and obviously $\delta_{s}=\left|e_{s}\right| \geq \rho_{T_{s}}$.

In the second case the endpoint $y_{s}$ belongs to $\overline{b_{s} c_{s}}$. Letting $\ell_{T_{s}}$ denote the perimeter of $T_{s}$, because $\sin \left(\widehat{b_{s}}\right)=$ $\frac{\left|T_{s}\right|}{\frac{1}{2}\left|a_{s} b_{s}\right|\left|b_{s} c_{s}\right|}=\frac{\frac{1}{2} \ell_{T_{s}} \rho_{T_{s}}}{\frac{1}{2}\left|a_{s} b_{s}\right|\left|b_{s} c_{s}\right|} \geq \frac{2 h_{T_{s}} \rho_{T_{s}}}{h_{T_{s}}^{2}}=\frac{2}{\sigma_{T_{s}}}$, we have $\delta_{s} \geq\left|x_{s} b_{s}\right| \sin \left(\widehat{b_{s}}\right) \geq \frac{1}{2}\left|e_{s}\right| \sin \left(\widehat{b_{s}}\right) \geq \frac{1}{\sigma_{T_{s}}} \rho_{T_{s}} \geq \frac{1}{\sigma_{\Omega}} \rho_{T_{s}}$.

In the third and last case $y_{s}$ belongs to $\overline{a_{s} c_{s}}$. Here there are two possibilities: if $y_{s}$ is closer to $c_{s}$ than to $a_{s}$, then $\delta_{s} \geq \frac{1}{2}\left|b_{s} c_{s}\right| \geq \frac{1}{2} \rho_{T_{s}}$. If on the other hand, $y_{s}$ is closer to $a_{s}$, then redefine $x_{s}$ to be $y_{s}, e_{s}$ to be $\overline{a_{s} c_{s}}$ and $T_{s}$ to be the other cell of $\mathcal{T}_{h}$ having $\overline{a_{s} c_{s}}$ as an edge. Repeat the previous arguments. One may change successively the point $x_{s}$, turning around $a_{s}$ until reaching one of the previous cases (recall that $s$ is a straight segment), see Fig. 3, right.

Conclude by noting that $\rho_{T_{s}} \geq \rho_{h} \geq \frac{h}{\sigma_{\Omega}}$, where we have used the uniform regularity of $\mathcal{T}_{h}$.

Lemma 3 With $\Pi_{h}$ defined by (14) and (15), if the pair $\left(\mathcal{T}_{h}, \mathcal{T}_{h, \lambda}\right)$ satisfies Hypotheses 1 and 2 , then Conditions 1 and 2 are satisfied.

Proof With the definition of $C_{s}$ given in (15), it is clear that Condition 2 is satisfied. Thus we have left to find an upper bound $C_{\Pi}$ on the continuity constants $C_{\Pi_{h}}$ of the operators $\Pi_{h}$. Since the support of $\phi_{s}$ is $\Delta_{a_{s}}$, we have, for each $p \in V_{\Omega}$, that

$$
\left\|\Pi_{h} p\right\|_{1, \Omega} \leq\left\|S Z_{h} p\right\|_{1, \Omega}+\left(\sum_{s \in \mathcal{T}_{h, \lambda}}\left|C_{s}\right|^{2}\left\|\phi_{s}\right\|_{1, \Delta_{a_{s}}}^{2}\right)^{\frac{1}{2}} .
$$

Estimates for the Scott-Zhang operator are well known, cf. [56] or [24, Lemma 1.130]:

$$
\begin{gathered}
\forall \ell, m, h, q ; \ell>\frac{1}{2} ; 0 \leq m \leq \min \{1, \ell\}, q \in H^{\ell}(\Omega), \\
\left\|\mathcal{S} Z_{h} q\right\|_{m, \Omega} \leq C_{\mathcal{S} Z}\|q\|_{\ell, \Omega}, \\
\forall \ell, m, h, T, q ; \frac{1}{2}<\ell \leq 2 ; 0 \leq m \leq \ell, T \in \mathcal{T}_{h}, q \in H^{\ell}\left(\Delta_{T}\right), \\
\left\|q-\mathcal{S} Z_{h} q\right\|_{m, T} \leq C_{\mathcal{S} Z} h^{\ell-m}|q|_{\ell, \Delta_{T}} .
\end{gathered}
$$

To estimate the second term of (16) we first calculate an upper bound for $\left\|\phi_{s}\right\|_{1, \Delta_{a_{s}}}^{2}$ : we have, $\forall s \in \mathcal{T}_{h, \lambda}$,

$$
\left\|\phi_{s}\right\|_{1, \Delta_{a_{s}}}^{2}=\sum_{T \in \mathcal{T}_{h}, T \subset \Delta_{a_{s}}}\left\|\phi_{s}\right\|_{1, T}^{2} .
$$


Using the technique of passing to a reference element and using the regularity of the mesh $\mathcal{T}_{h}$ we obtain that there is a constant $C_{\widehat{T}}$ independent of $h$ such that for each $s \in \mathcal{T}_{h, \lambda}$, and for each $T \in \mathcal{T}_{h}$ with $T \subset \Delta_{a_{s}}$,

$$
\left\|\phi_{s}\right\|_{1, T}^{2} \leq \sigma_{T} C_{\widehat{T}} \quad \text { and } \quad\left\|\phi_{s}\right\|_{1, \Delta_{a_{s}}}^{2} \leq \eta \sigma_{\Omega} C_{\widehat{T}}
$$

where $\eta$ is the maximum number of cells of $\mathcal{T}_{h}$ meeting at a vertex of $\mathcal{T}_{h}$. (Such a maximum exists because the grid, belonging to a regular family, has a minimum angle size.) To control the constants $C_{s}, s \in \mathcal{T}_{h, \lambda}$, we use Lemma 2 to obtain a lower bound for $\left.\int_{s} \phi_{s}\right|_{s}$, and we establish an upper bound for $\left.\int_{s}\left(\mathcal{S} Z_{h} p-p\right)\right|_{s}$, using the Cauchy-Schwarz inequality, Lemma 1 and the estimates for the Scott-Zhang interpolation operator:

$$
\begin{aligned}
\left.\int_{s}\left(\mathcal{S} Z_{h} p-p\right)\right|_{s} \leq|s|^{\frac{1}{2}}\left\|\left.\left(\mathcal{S} Z_{h} p-p\right)\right|_{s}\right\|_{0, s} \\
\leq C_{\tau}^{\frac{1}{2}}|s|^{\frac{1}{2}}\left\{\sum_{T \in \mathcal{T}_{h} ; T \cap s \neq \emptyset} \sigma_{T}\right. \\
\left.\quad\left(h_{T}^{-1}\left\|\mathcal{S} Z_{h} p-p\right\|_{0, T}^{2}+h_{T}\left|\mathcal{S} Z_{h} p-p\right|_{1, T}^{2}\right)\right\}^{\frac{1}{2}} \\
\leq C_{\tau}^{\frac{1}{2}}|s|^{\frac{1}{2}} \sigma_{\Omega}^{\frac{1}{2}}\left\{\sum_{T \in \mathcal{T}_{h} ; T \cap s \neq \emptyset} 2 C_{\mathcal{S} Z}^{2} h|p|_{1, \Delta_{T}}^{2}\right\}^{\frac{1}{2}} \\
\leq \sqrt{2} C_{\mathcal{S} Z} h^{\frac{1}{2}} C_{\tau}^{\frac{1}{2}}|s|^{\frac{1}{2}} \sigma_{\Omega}^{\frac{1}{2}}\left\{\sum_{T \in \mathcal{T}_{h} ; T \cap s \neq \emptyset}|p|_{1, \Delta_{T}}^{2}\right\}^{\frac{1}{2}}
\end{aligned}
$$

We thus have

$$
\begin{aligned}
& \sum_{s \in \mathcal{T}_{h, \lambda}}\left|C_{s}\right|^{2}\left\|\phi_{s}\right\|_{1, \Delta_{a_{s}}}^{2} \leq \eta \sigma_{\Omega} C_{\widehat{T}} \sum_{s \in \mathcal{T}_{h, \lambda}}\left|C_{s}\right|^{2} \\
& \leq \eta \sigma_{\Omega} C_{\widehat{T}} \sum_{s \in \mathcal{T}_{h, \lambda}} \frac{1}{C_{\phi}^{2} h^{2}}\left\{\left.\int_{s}\left(\mathcal{S} Z_{h} p-p\right)\right|_{s}\right\}^{2} \\
& \leq \eta \sigma_{\Omega}^{2} C_{\widehat{T}} \frac{1}{C_{\phi}^{2} h^{2}} 2 C_{\mathcal{S} Z}^{2} h C_{\tau} \sum_{s \in \mathcal{T}_{h, \lambda}}|s|\left(\sum_{T \in \mathcal{T}_{h} ; T \cap s \neq \emptyset}|p|_{1, \Delta_{T}}^{2}\right) \\
& \leq \eta \sigma_{\Omega}^{2} C_{\widehat{T}} \frac{1}{C_{\phi}^{2} h^{2}} 2 C_{\mathcal{S} Z}^{2} h C_{\tau} L h \sum_{s \in \mathcal{T}_{h, \lambda}}\left(\sum_{T \in \mathcal{T}_{h} ; T \cap s \neq \emptyset}|p|_{1, \Delta_{T}}^{2}\right) \\
& \leq 2 \eta \sigma_{\Omega}^{2} C_{\widehat{T}} \frac{1}{C_{\phi}^{2}} C_{\mathcal{S} Z}^{2} C_{\tau} L \tilde{\eta}|p|_{1, \Omega}^{2} \\
& =C_{1}^{2}|p|_{1, \Omega}^{2},
\end{aligned}
$$

where $\tilde{\eta}=\sup _{T \in \mathcal{T}_{h}} \tilde{\eta}_{T}$ with $\tilde{\eta}_{T}$ the cardinality of $\left\{\left(s, T^{\prime}\right) \in \mathcal{T}_{h, \lambda} \times \mathcal{T}_{h}: s \cap T^{\prime} \neq \emptyset\right.$ and $\left.T \subset \Delta_{T^{\prime}}\right\}$. Thus one obtains

$\left\|\Pi_{h} p\right\|_{1, \Omega} \leq\left(C_{\mathcal{S Z}}+C_{1}\right)\|p\|_{1, \Omega}$,

and Condition 1 is satisfied with $C_{\Pi}=C_{\mathcal{S Z}}+C_{1}$.
Remark 5 We have supposed that $\mathcal{T}_{h}$ is uniformly regular. In fact we only need uniform regularity in a narrow strip containing $\gamma$.

In light of Brezzi's theory for mixed methods [14,20, 53 , the following theorem is an immediate consequence of Lemma 3:

Theorem 2 Assume that $\Lambda_{h}$ is defined by (12) and that Hypotheses 1 and 2 concerning the meshes $\mathcal{T}_{h}$ and $\mathcal{T}_{h, \lambda}$ hold. Then there exists a unique solution $\left(p_{h}, p_{\gamma, h}\right.$; $\left.\lambda_{h}\right) \in \boldsymbol{V}_{h} \times \Lambda_{h}$ to the discrete, Lagrange-multiplier, fracture problem (11). Moreover, there exists a constant $C$ independent of $h, h_{\lambda}$ and $h_{\gamma}$ such that

$$
\begin{aligned}
& \left\|p-p_{h}\right\|_{1, \Omega}+\left\|p_{\gamma}-p_{\gamma, h}\right\|_{1, \gamma}+\left\|\lambda-\lambda_{h}\right\|_{-\frac{1}{2}, \gamma} \\
& \leq C\left(\inf _{q_{h} \in V_{h, \Omega}}\left\|p-q_{h}\right\|_{1, \Omega}+\inf _{q_{\gamma, h} \in V_{h, \gamma}}\left\|p_{\gamma}-q_{\gamma, h}\right\|_{1, \gamma}\right. \\
& \left.+\inf _{\mu_{h} \in \Lambda_{h}}\left\|\lambda-\mu_{h}\right\|_{-\frac{1}{2}, \gamma}\right) .
\end{aligned}
$$

To obtain a convergence estimate, it remains to use approximation results. These are standard for $p$ and $p_{\gamma}$. For $\lambda$, we can use the result of [34, Lemma 7]: there exists $C_{L}$ independent of $h_{\lambda}$ such that for all $\mu \in H^{\tilde{\alpha}}(\gamma)$ for $\tilde{\alpha} \in\left\{0, \frac{1}{2}\right\}$ we have

$$
\inf _{\mu_{h} \in \Lambda_{h}}\left\|\mu-\mu_{h}\right\|_{-\frac{1}{2}, \gamma} \leq C_{L} h_{\lambda}^{\frac{1}{2}+\tilde{\alpha}} \inf _{\mu_{h} \in \Lambda_{h}}\left\|\mu-\mu_{h}\right\|_{\tilde{\alpha}, \gamma} .
$$

Theorem 3 Assume that the hypotheses of Theorem 2 hold. Let $\left(p, p_{\gamma} ; \lambda\right) \in \boldsymbol{V} \times \Lambda$ be the solution to Problem (9), and $\left(p_{h}, p_{\gamma, h} ; \lambda_{h}\right) \in \boldsymbol{V}_{h} \times \Lambda_{h}$ be the solution to Problem (11). Assume that $\left(p, p_{\gamma}\right)$ belongs to $H^{\alpha}(\Omega) \times$ $H^{2}(\gamma)$ for an $\alpha \in\left[\frac{3}{2}-\varepsilon, 2\right](\varepsilon>0)$, and that $\lambda$ belongs to $H^{\tilde{\alpha}}(\gamma)$ for some $\tilde{\alpha} \in\left[0, \frac{1}{2}\right]$. Then, there exists a constant $C$ independent of $h, h_{\lambda}$ and $h_{\gamma}$ such that

$$
\begin{aligned}
& \left\|p-p_{h}\right\|_{1, \Omega}+\left\|p_{\gamma}-p_{\gamma, h}\right\|_{1, \gamma}+\left\|\lambda-\lambda_{h}\right\|_{-\frac{1}{2}, \gamma} \\
& \leq C\left(h^{\alpha-1}\|p\|_{\alpha, \Omega}+h_{\gamma}\left\|p_{\gamma}\right\|_{2, \gamma}+h_{\lambda}^{\frac{1}{2}+\tilde{\alpha}}\|\lambda\|_{\tilde{\alpha}, \gamma}\right) .
\end{aligned}
$$

Thus, depending on the regularity of the continuous solution, one expects a convergence rate of order between $h^{\frac{1}{2}-\varepsilon}$ and $h$. In all of the numerical experiments that we have examined we obtained an order of at least $h$ for the variables $p_{h}$ and $p_{\gamma, h}$ and an order between $h^{\frac{1}{2}}$ and $h$ for $\lambda_{h}$ :cf. Fig. 5, Fig. 7 and Fig. 10 .

3.3 An alternative Lagrange multiplier space with continuous, piecewise-linear multipliers

The study in this subsection is motivated by the idea that for some alternative, discrete, Lagrange-multiplier space it might be possible to use a relation between the Lagrange multiplier space and the fracture pressure space to establish a discrete inf-sup condition. Here 
we consider the simplest possible such case: the case in which the spaces $V_{h, \Omega}$ and $V_{h, \gamma}$ are still defined by (10), but the fracture meshes are equal, $\mathcal{T}_{h, \lambda}=\mathcal{T}_{h, \gamma}$, and indeed (12) is replaced by

$$
\Lambda_{h}=V_{h, \gamma}
$$

Proposition 1 Assume that $\Lambda_{h}$ is defined by (19) and that $\mathcal{T}_{h, \lambda}=\mathcal{T}_{h, \gamma}$ is uniformly regular. Then there exists a unique solution $\left(p_{h}, p_{\gamma, h} ; \lambda_{h}\right) \in \boldsymbol{V}_{h} \times \Lambda_{h}$ to the discrete, Lagrange-multiplier, fracture problem (11).

Proof We show that the discrete inf-sup condition is satisfied by the operator $b(\cdot, \cdot ; \cdot)$ on $\boldsymbol{V}_{h} \times \Lambda_{h}$, using the norm $\|\cdot\|_{\boldsymbol{V}}$ for $\boldsymbol{V}_{h}$ and for $\Lambda_{h}$ the discrete norm defined for $\mu_{h} \in \Lambda_{h}$ by

$$
\left\|\mu_{h}\right\|_{\Lambda_{h}}=\left\|\mu_{h}\right\|_{-\frac{1}{2}, h_{\lambda}, \gamma}=h_{\lambda}^{\frac{1}{2}}\left\|\mu_{h}\right\|_{0, \gamma} .
$$

Let $\lambda_{h} \in \Lambda_{h}$. As $\Lambda_{h}=V_{h, \gamma}$, we take $r_{h, \gamma}=-\lambda_{h}$ and $r_{h}=0$. Then we have

$$
b\left(r_{h}, r_{h, \gamma} ; \lambda_{h}\right)=b\left(0,-\lambda_{h} ; \lambda_{h}\right)=\left\|\lambda_{h}\right\|_{0, \gamma}^{2}
$$

Using the uniform regularity of the mesh $\mathcal{T}_{h, \lambda}=\mathcal{T}_{h, \gamma}$, and using an inverse inequality [24, Corollary 1.141, page 76 or Remark 1.143 (i), page 77], there is a constant $\hat{\theta}$ such that

$$
\begin{aligned}
\left\|\left(r_{h}, r_{h, \gamma}\right)\right\|_{\boldsymbol{V}}\left\|\lambda_{h}\right\|_{\Lambda_{h}} & =\left\|\lambda_{h}\right\|_{1, \gamma}\left\|\lambda_{h}\right\|_{-\frac{1}{2}, h_{\lambda}, \gamma} \\
& =\left\|\lambda_{h}\right\|_{1, \gamma} h_{\lambda}^{\frac{1}{2}}\left\|\lambda_{h}\right\|_{0, \gamma} \\
& \leq \hat{\theta} h_{\lambda}^{-\frac{1}{2}}\left\|\lambda_{h}\right\|_{0, \gamma}^{2} \\
& \leq \hat{\theta} h_{\lambda}^{-\frac{1}{2}} b\left(r_{h}, r_{h, \gamma} ; \lambda_{h}\right) .
\end{aligned}
$$

Thus the discrete inf-sup condition is satisfied with a constant $\theta_{h}=\hat{\theta}^{-1} h_{\lambda}^{\frac{1}{2}}$ which is not independent of $h_{\lambda}$.

Remark 6 Note that there is no compatibility condition on the meshes $\mathcal{T}_{h}$ and $\mathcal{T}_{h, \lambda}=\mathcal{T}_{h, \gamma}$ in the proof, neither does $\mathcal{T}_{h}$ need to be uniformly regular. In particular there is no hypothesis on the respective mesh sizes $h$ and $h_{\lambda}=h_{\gamma}$. However with this choice of $\Lambda_{h}$, the discrete inf-sup condition is not uniform in $h_{\lambda}$, so one can assure a unique solution but not convergence. We remark that the numerical results of Section 4 show good convergence rates even for this choice of $\Lambda_{h} ; c f$. Fig. 5, Fig. 7 and Fig. 11.

\section{Numerical results}

The emphasis of this section is on numerical experiments using the two different discretizations presented in Section 3. In particular the mesh size of the discrete multipliers $h_{\lambda}$ and its impact on accuracy and convergence will be addressed. We recall that the two discretizations of the continuous problem (9) vary only in the way the Lagrange multiplier is defined. By $\left.\lambda_{h}\right|_{s} \in$
$\mathbb{P}^{0}(s), c f$. (12), we refer to the discretization of Section 3.2 and by $\left.\lambda_{h}\right|_{s} \in \mathbb{P}^{1}(s)$, cf. (19), to the discretization of Section 3.3. The latter implies that $h_{\lambda}=h_{\gamma}$. In the former case we consider $h_{\lambda} \approx 2 h$, in order to show that the condition $h_{\lambda}>3 h$ (see Remark 1) is sufficient but probably too restrictive in practice. Due to the higher permeability in the fracture, which may demand higher accuracy at the fracture interface, in all cases we have taken $h_{\gamma} \leq h$. With the above choices of $h_{\lambda}$ we ensure that $h_{\lambda} \geq \min \left\{h, h_{\gamma}\right\}$, to prevent a poorly conditioned system matrix.

We analyze the performance of the proposed method by means of several test cases of different complexity. Based on an oblique fracture extending up to the boundaries of the matrix domain and an internal fracture with a junction and with fracture tips in the interior of the matrix, we study the numerical convergence of the method in Section 4.1 and Section 4.2, respectively. These examples are followed by more complex test cases with a regular fracture network in Section 4.3 and a more realistic benchmark problem in Section 4.4. The accuracy and convergence are validated by means of a comparison with other methods in this field, [27].

As the fracture interface is generally not aligned with the matrix mesh, we use a sufficiently fine reference solution for the computation of the error in all examples. In the simplified case of a conforming fracture, i.e. aligned with the matrix mesh, we obtain optimal rates of convergence in the matrix as well as in the fracture (results not shown here, see [42]; cf. also [43]). A direct solver was used to solve the linear systems.

\subsection{Case 1: an oblique fracture}

The first setup is a two-dimensional, square domain $\Omega:=[0,1]^{2}$ with homogeneous Neumann conditions on the horizontal boundaries and nonhomogeneous Dirichlet conditions on the vertical boundaries ( $p=1$ on the left and $p=4$ on the right). An oblique fracture $\gamma$ with $\mathbf{K}_{\gamma}=10 \mathbf{I}_{\gamma}, \mathbf{K}=\mathbf{I}$, extends from the left to the right part of the horizontal boundaries of the matrix domain $\Omega$ with Dirichlet boundary values $p_{\gamma}=1$ on the lower tip and $p_{\gamma}=4$ on the upper tip. The test case and the pressure distribution is shown in Fig. 4. Because of the geometry of the setup the oblique fracture mesh is not aligned with the rectangular elements of the matrix grid (nonconforming). Fig. 5 displays the numerical convergence analysis of the primary variables in different norms for $h \in\{1 / 8,1 / 16, \ldots, 1 / 128\}$ based on a reference solution computed with a resolution of $h=1 / 512$. The mesh size of the fracture $h_{\gamma}$ is always half of the matrix mesh size, i.e. $h_{\gamma} \approx h / 2$.

The results in Fig. 5 are in accordance with or better than the theoretical findings: the $H^{1}$ errors for fracture and matrix pressures converge linearly independent of the utilized discretization of the Lagrange multiplier and the Lagrange multiplier converges with rates up to 


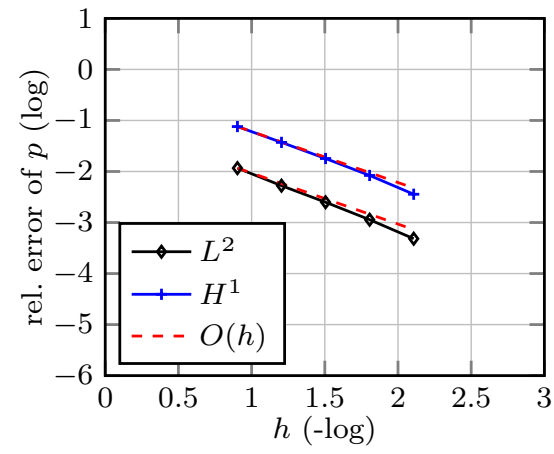

(a) Error of $p\left(h_{\lambda} \approx 2 h,\left.\lambda_{h}\right|_{s} \in \mathbb{P}^{0}(s)\right)$

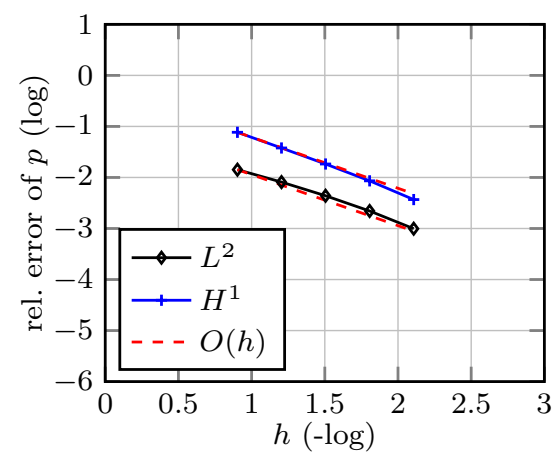

(d) Error of $p\left(h_{\lambda}=h_{\gamma},\left.\lambda_{h}\right|_{s} \in \mathbb{P}^{1}(s)\right)$

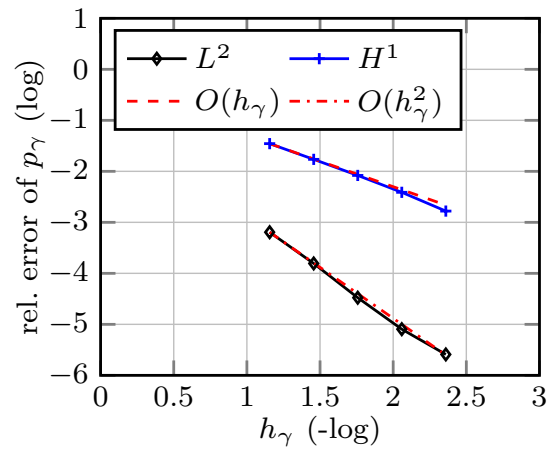

(b) Error of $p_{\gamma}\left(h_{\lambda} \approx 2 h,\left.\lambda_{h}\right|_{s} \in \mathbb{P}^{0}(s)\right)$

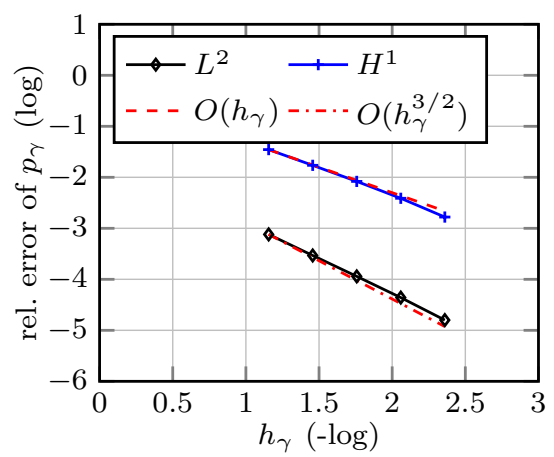

(e) Error of $p_{\gamma}\left(h_{\lambda}=h_{\gamma},\left.\lambda_{h}\right|_{s} \in \mathbb{P}^{1}(s)\right)$

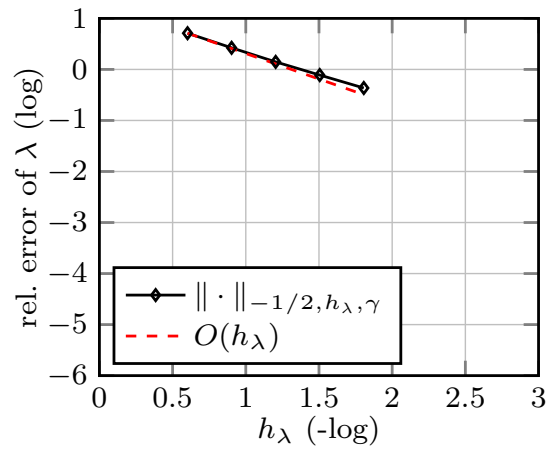

(c) Error of $\lambda\left(h_{\lambda} \approx 2 h,\left.\lambda_{h}\right|_{s} \in \mathbb{P}^{0}(s)\right)$

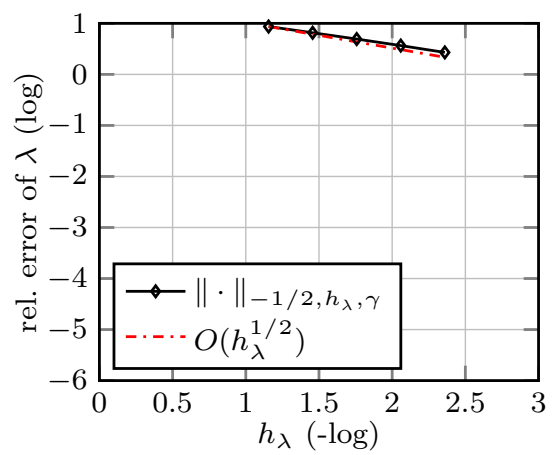

(f) Error of $\lambda\left(h_{\lambda}=h_{\gamma},\left.\lambda_{h}\right|_{s} \in \mathbb{P}^{1}(s)\right)$

Fig. 5 Case 1 (an oblique fracture): Convergence of matrix pressue $p$, fracture pressure $p_{\gamma}$ and Lagrange multiplier $\lambda$ for $h_{\lambda} \approx 2 h,\left.\lambda_{h}\right|_{s} \in \mathbb{P}^{0}(s)$ (above) and for $h_{\lambda}=h_{\gamma},\left.\lambda_{h}\right|_{s} \in \mathbb{P}^{1}(s)$ (below) depending on the mesh size.

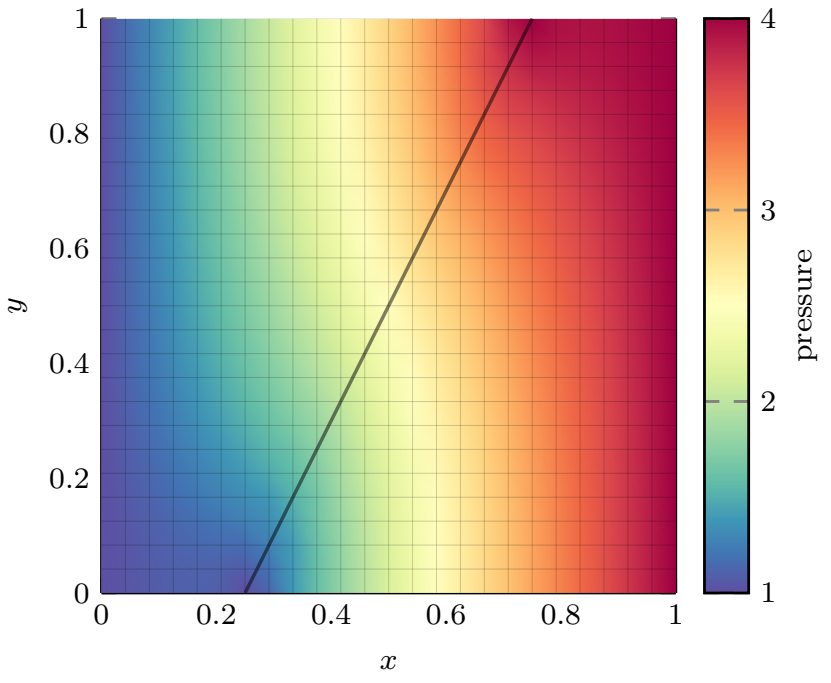

Fig. 4 Case 1 (an oblique fracture): Pressure distribution of a simulation with $h=1 / 24, h_{\lambda}=h_{\gamma}=1 / 48,\left.\lambda_{h}\right|_{s} \in \mathbb{P}^{1}(s)$.

$O\left(h_{\lambda}\right)$ in the discrete norm $\|\cdot\|_{-\frac{1}{2}, h_{\lambda}, \gamma}$ defined in $(20)$. The Lagrange multiplier error remains at quite high levels, but this does not degrade the error for $p$ or $p_{\gamma}$ which are the more relevant physical unknowns. In the $L^{2}$ norm, the errors for the matrix pressure converge lin- early, and the errors for the fracture pressure converge with rates between $O\left(h_{\gamma}^{\frac{3}{2}}\right)$ and $O\left(h_{\gamma}^{2}\right)$. Additional numerical experiments (not presented here) showed that it is possible to use a more refined fracture mesh, e.g. $h_{\gamma} \approx h / 10$, with conclusions in no way significantly different from those above. The only difference is an improvement in the fracture pressure error.

We conclude that in the present test case with an oblique fracture the choice of the ansatz functions of the Lagrange multiplier does not affect the rates of convergence and the errors significantly. The major difference in Fig. 5 stems from the choice of the mesh sizes.

Remark 7 When $h_{\lambda}$ is too small (typically $h_{\lambda}<h / 2$ ), Hypotheses 1 and 2 are not satisfied by far, and problem (11) is not stable. This is characterized by solvability issues of the linear system (at least a very poor conditionning of the matrix), [42].

\subsection{Case 2: a y-shaped fracture}

The next setup rests upon a slightly different fracturematrix scenario with fracture tips ending within the matrix domain $\Omega$. In contrast to the test case of the previous Section $4.1 \mathrm{a}$ y-shaped fracture splitting into two parts, is located in the center of the domain. We 


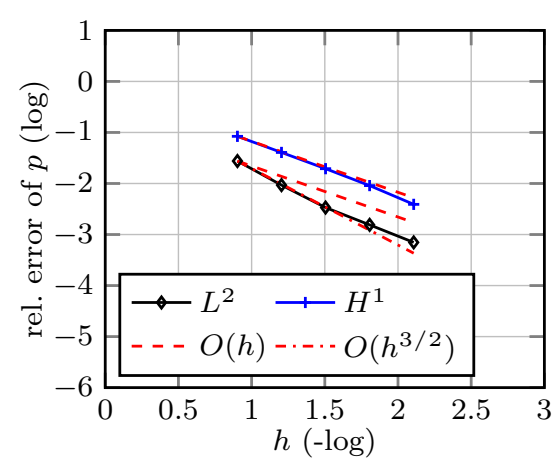

(a) Error of $p\left(h_{\lambda} \approx 2 h,\left.\lambda_{h}\right|_{s} \in \mathbb{P}^{0}(s)\right)$

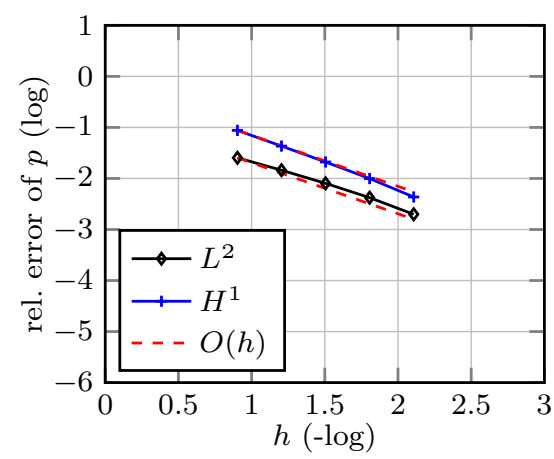

(d) Error of $p\left(h_{\lambda}=h_{\gamma},\left.\lambda_{h}\right|_{s} \in \mathbb{P}^{1}(s)\right)$

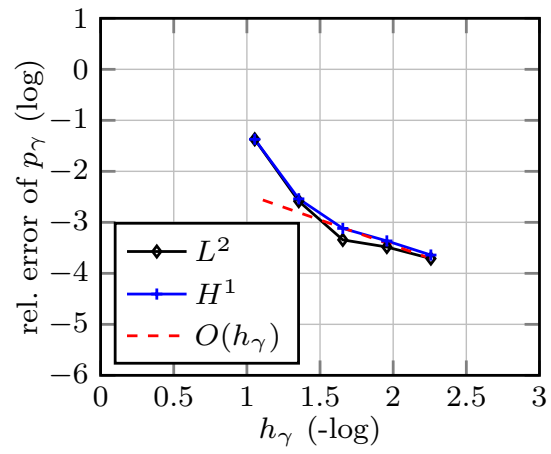

(b) Error of $p_{\gamma}\left(h_{\lambda} \approx 2 h,\left.\lambda_{h}\right|_{s} \in \mathbb{P}^{0}(s)\right)$

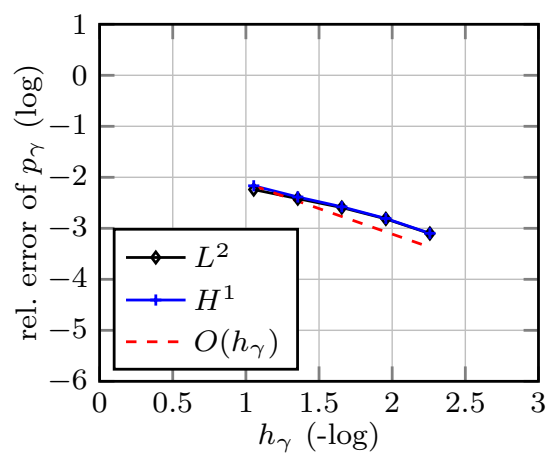

(e) Error of $p_{\gamma}\left(h_{\lambda}=h_{\gamma},\left.\lambda_{h}\right|_{s} \in \mathbb{P}^{1}(s)\right)$

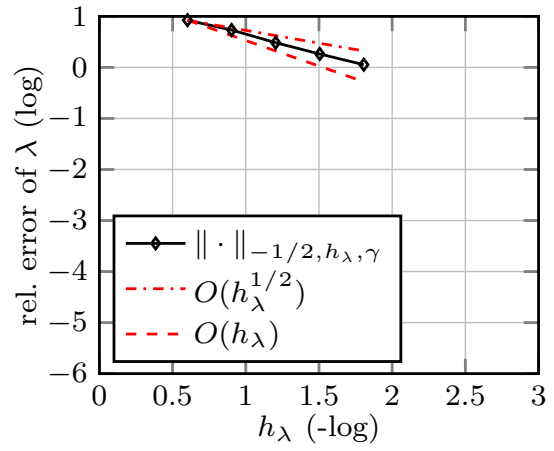

(c) Error of $\lambda\left(h_{\lambda} \approx 2 h,\left.\lambda_{h}\right|_{s} \in \mathbb{P}^{0}(s)\right)$

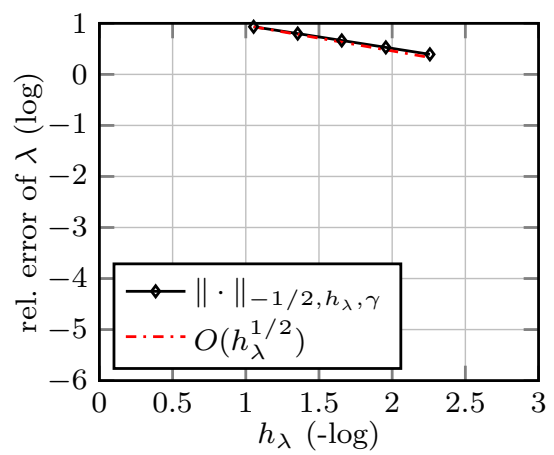

(f) Error of $\lambda\left(h_{\lambda}=h_{\gamma},\left.\lambda_{h}\right|_{s} \in \mathbb{P}^{1}(s)\right)$

Fig. 7 Case 2 (a y-shaped fracture): Convergence of matrix pressure $p$, fracture pressure $p_{\gamma}$ and Lagrange multiplier $\lambda$ for $h_{\lambda} \approx 2 h,\left.\lambda_{h}\right|_{s} \in \mathbb{P}^{0}(s)$ (above) and for $h_{\lambda}=h_{\gamma},\left.\lambda_{h}\right|_{s} \in \mathbb{P}^{1}(s)$ (below) depending on the mesh size.

impose no flow conditions at the fracture tips. The test case and the pressure distribution is shown in Fig. 6 . The numerical convergence study in different norms is

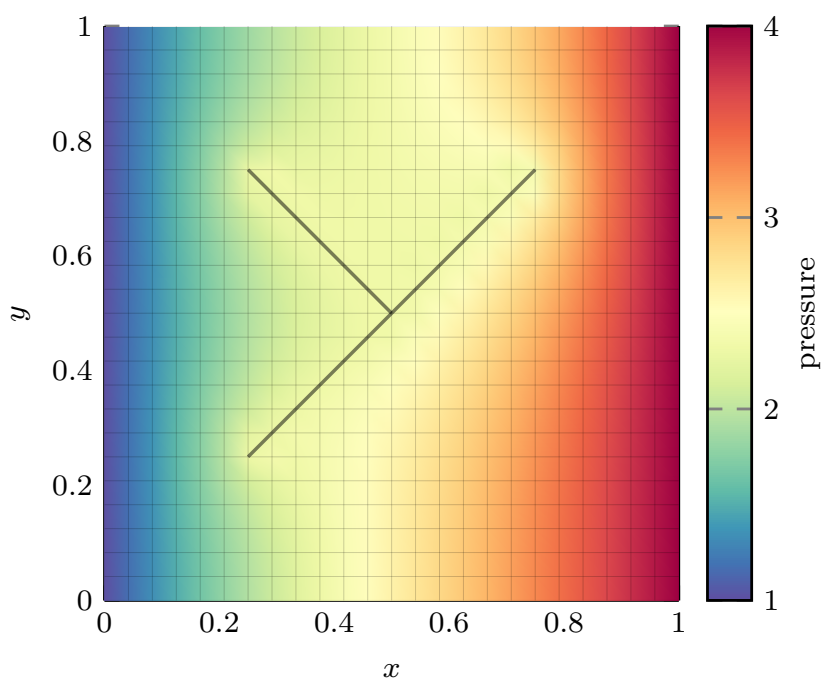

Fig. 6 Case 2 (a y-shaped fracture): Pressure distribution of a simulation with $h=1 / 24, h_{\lambda}=h_{\gamma}=1 / 48,\left.\lambda_{h}\right|_{s} \in \mathbb{P}^{1}(s)$.

displayed in Fig. 7 for $h \in\{1 / 8,1 / 16, \ldots, 1 / 128\}$ based on a reference solution computed with resolution $h=$ $1 / 512$.

Apart from a small kink in Fig. 7(b) the linear rate of convergence of the $H^{1}$ error observed in the previous example ( $c f$. Fig. 5) is maintained. The $L^{2}$ errors have approximately linear convergence with slighly improved rates of the matrix in the case $h_{\lambda} \approx 2 h,\left.\lambda_{h}\right|_{s} \in \mathbb{P}^{0}(s)$. It is worth noticing that the difference of $L^{2}$ and $H^{1}$ error in the fracture is marginal. The absolute value of the $L^{2}$ errors are higher compared to those of Fig. 5. The errors of the Lagrange multiplier are in the range of $O\left(h_{\lambda}^{1 / 2}\right)$ and $O\left(h_{\lambda}^{3 / 4}\right)$. Again additional numerical experiments show that it is possible to use a more refined fracture mesh, e.g. $h_{\gamma} \approx h / 10$, with roughly the same conclusions.

We conclude that in this case of a splitting and internal fracture the choice of the ansatz function of the discrete Lagrange multipliers has a stronger impact on the convergence rates. Nonetheless the numerical experiments indicate the overall convergence. The junction of the fracture lines and the fracture tips ending within the matrix domain may additionally reduce the regularity of the solution. This may explain why the choice of the ratio of the involved mesh sizes becomes more important. 


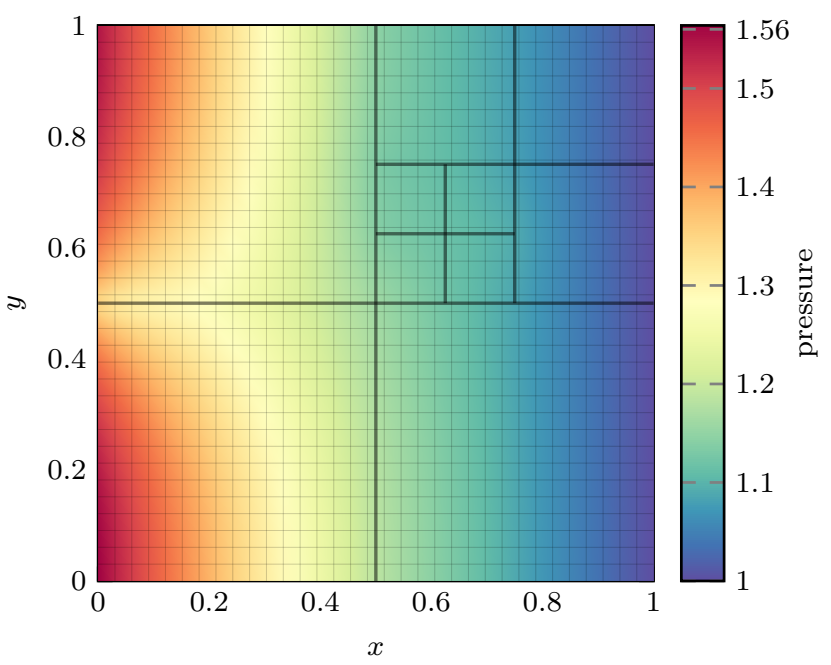

Fig. 8 Case 3 (a fracture network): Pressure distribution of simulation with $h=1 / 33, h_{\gamma}=1 / 32, h_{\lambda} \approx 2 h,\left.\lambda_{h}\right|_{s} \in \mathbb{P}^{0}(s)$. The fracture network is shown in black.

\subsection{Case 3: a fracture network}

The third setup is a more complex test case with a regular fracture network presented in [32] but with boundary conditions slightly modified (in accordance with [27]). On the horizontal boundaries homogeneous Neumann conditions are imposed. Moreover we impose nonhomogeneous Neumann conditions (inflow) on the left and nonhomogeneous Dirichlet boundary conditions on the right. All fractures of the test case have the uniform aperture $d=10^{-4}$. The matrix domain is characterized by a permeability of $\mathbf{K}=\mathbf{I}$ and the fracture by a permeability of $\mathbf{K}_{\gamma}=\mathbf{I}_{\gamma}$. Note that the aperture of the fracture is incorporated in the value of $\mathbf{K}_{\gamma}$ and the inflow condition $\mathbf{u}_{\gamma} \cdot \mathbf{n}_{\gamma}$ on the left boundary. The resulting pressure distribution of a simulation with nonconforming configuration and $h=1 / 33, h_{\gamma}=1 / 32, h_{\lambda} \approx 2 h$, $\left.\lambda_{h}\right|_{s} \in \mathbb{P}^{0}(s)$ is shown in Fig. 8 .

Based on the benchmark study [27] we compare the proposed Lagrange-multiplier method with several other available methods in the field of single-phase flow in fractured porous media. The reference solution used here is computed with a mimetic finite difference method [21] using a two-dimensional grid in the fracture and the matrix domain. The interested reader is referred to [27] for further details of the different methods. Fig. 9 displays the comparison of the pressure profile at $y=0.7$ and $x=0.5$, where the proposed method of this work is denoted by LM-FEM, (Lagrange multiplier - finite element method). Fig. 9 shows that the distribution of the pressure is in good agreement with the other methods. With 1089 rectangular elements in the matrix and 112 segments in the fracture the simulation of the Lagrange multiplier method is in the same range of numbers of elements as the other methods. Similarly, the $L^{2}$ error
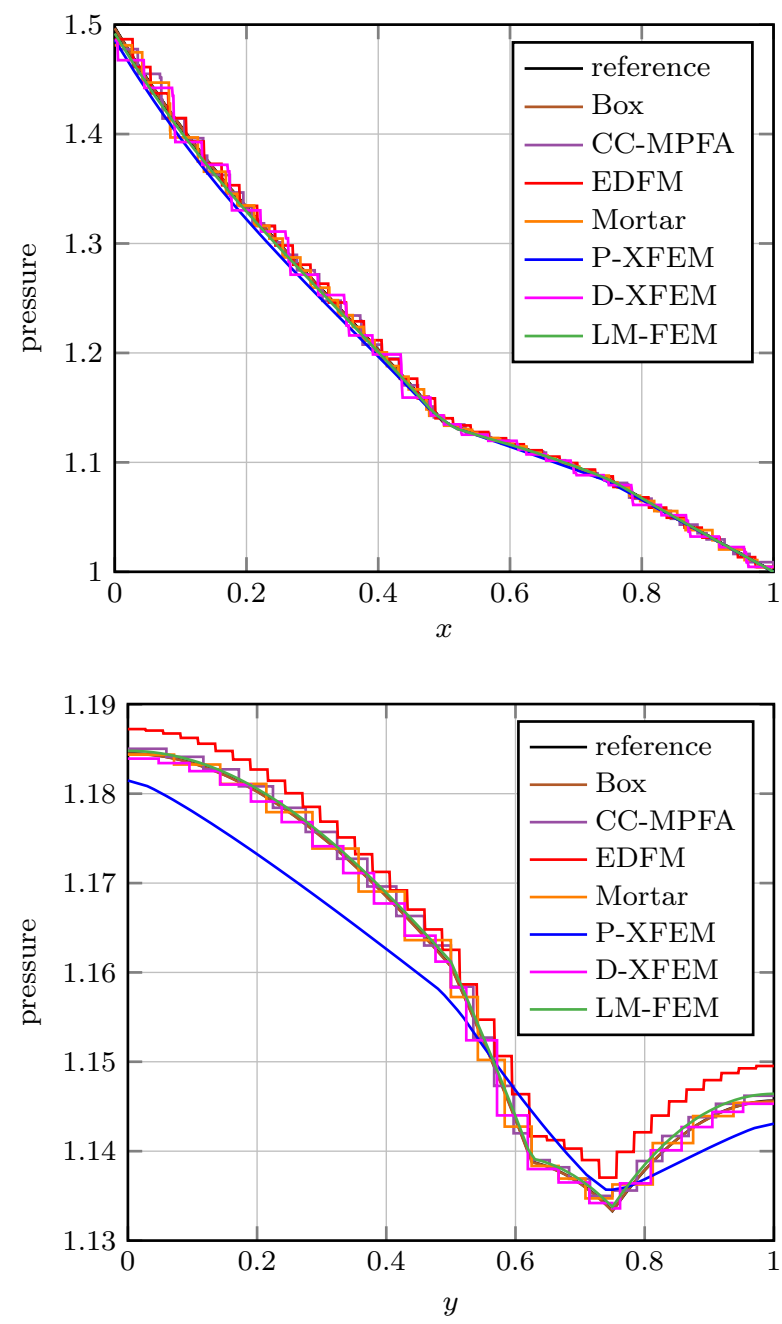

Fig. 9 Case 3 (a fracture network): Comparison with other methods, $c f$. [27], at $y=0.7$ (above) and $x=0.5$ (below). Lagrange multiplier method (with $\left.\lambda_{h}\right|_{s} \in \mathbb{P}^{0}(s), h=1 / 33, h_{\gamma}=$ $1 / 32, h_{\lambda} \approx 2 h$ and nonconforming grid) denoted by LMFEM.

in the matrix $\mathrm{err}_{m}=1.0 \cdot 10^{-2}$ and in the fracture $e r r_{\gamma}=6.5 \cdot 10^{-3}$ is in the same range.

Building on this configuration the meshes are consecutively refined three times by a factor of two $(h \in$ $\{1 / 33,1 / 65,1 / 129,1 / 257\})$ in order to investigate the convergence in a similar way as proposed in [27]. The resulting convergence study of both, matrix and fracture pressure, is illustrated in Fig. 10. Again the Lagrange multiplier method is characterized by a similar convergence behavior as the other methods of the benchmark, with linear convergence rates in the matrix and the fracture. Additonal numerical experiments indicated linear rates of convergence also for different discretizations such as $\left.\lambda_{h}\right|_{s} \in \mathbb{P}^{1}(s), h_{\lambda}=h_{\gamma}$ or a refined fracture mesh as long as the mesh of the multiplier is not chosen too small compared to the matrix mesh, e.g. $h_{\lambda} \geq h / 2$. 

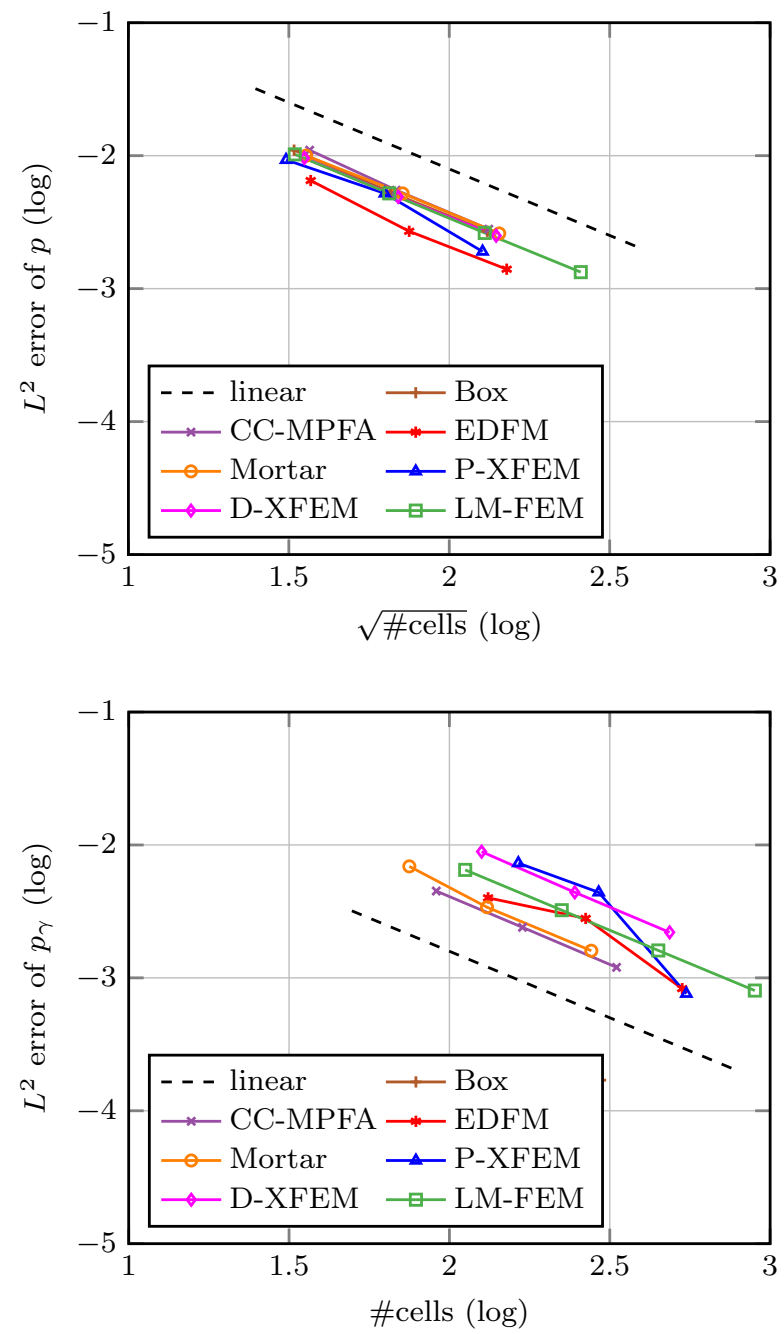

Fig. 10 Case 3 (a fracture network): Convergence of matrix pressure $p$ (above) and fracture pressure $p_{\gamma}$ (below) for $h_{\lambda} \approx 2 h,\left.\lambda_{h}\right|_{s} \in \mathbb{P}^{0}(s)$ depending on the number of elements compared to other methods, $c f$. [27]. Lagrange multiplier method denoted by LM-FEM.

The numerical results demonstrate that in practice the mesh size of the Lagrange multiplier can be chosen smaller than the theoretical results of Section 3 suggest. Moreover the results show that the multiplier method is able to compete with other DFM models in the field.

\subsection{Case 4: the hydrocoin benchmark}

The last setup is a more realistic flow problem of the international hydrocoin project [57]. The benchmark accounts for the morphology of the geological formation and is characterized by the piezometric head and hydraulic conductivities. It comprises two conductive and intersecting fractures with different aperture, $d_{1} \approx$ $7.07 \mathrm{~m}$ and $d_{2} \approx 14.76 \mathrm{~m}$, and uniform hydraulic conductivity $10^{-6} \mathrm{~m} / \mathrm{s}$. The surrounding matrix rock has an isotropic hydraulic conductivity of $10^{-8} \mathrm{~m} / \mathrm{s}$. The surface elevation represents the Dirichlet boundary condition at the top of the domain. No-flow Neumann boundary conditions are imposed on the other boundaries. Note that we slightly modified the original domain as in [27] in order to preserve the comparability between different fracture models. The plot at the top of Fig. 11 shows the distribution of the piezometric head of a simulation with $h \approx 34 m, h_{\gamma}=h_{\lambda} \approx h / 2,\left.\lambda_{h}\right|_{s} \in \mathbb{P}^{1}(s)$.
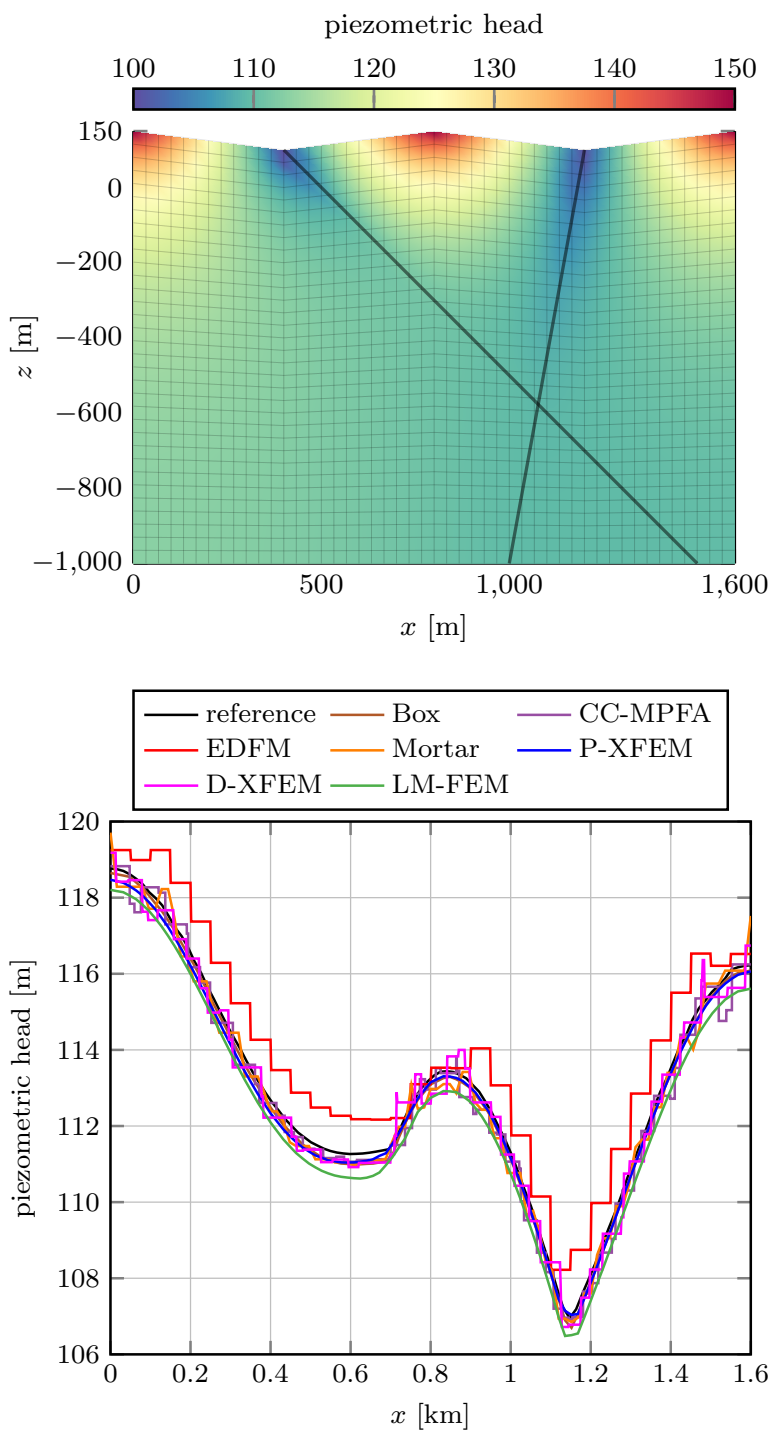

Fig. 11 Case 4 (the hydrocoin benchmark): Nonconforming simulation with $h \approx 34 m, h_{\gamma}=h_{\lambda} \approx h / 2,\left.\lambda_{h}\right|_{s} \in \mathbb{P}^{1}(s)$ (above) and comparison with other methods, cf. [27], at $z=$ $-200 m$ (below). Lagrange multiplier method denoted by LMFEM.

A comparison with other fracture models at a depth of $200 \mathrm{~m}$ based on [27] is shown in the plot at the bottom of Fig. 11. The reference solution used here is again computed with a mimetic finite difference method [21] using a two-dimensional grid in the fracture and in the 
matrix domain. Fig. 11 indicates that the approximation with the proposed method stays within the range of the compared distributions of the hydraulic head. The global error in the matrix $\mathrm{err}_{m}=1.6 \cdot 10^{-2}$ and the fracture error $e r r_{\gamma}=1.3 \cdot 10^{-2}$ are slightly higher than those of the other methods. Additional simulations with piecewise constant multipliers $\left.\lambda_{h}\right|_{s} \in \mathbb{P}^{0}(s)$ showed that the errors increase rapidly with the coarsening of the multiplier mesh. However a more refined fracture mesh does not affect the results significantly.

A more detailed convergence study was not performed in this case since the large width of the fractures raise the question if the equidimensional mimetic finite difference solution is a precise reference solution to reduced models of co-dimension one.

\section{Conclusion}

We presented a novel approach to modeling single-phase, single-component, Darcy flow in fractured porous media based on the use of a Lagrange multiplier, which couples the flow in the fracture with that in the surrounding matrix, and showed that there exists a unique solution to the primal formulation of the continuous problem. In particular this method allows for the use of a mesh in the matrix domain that is not aligned with the fracture. In order to approximate the fracture and the matrix pressure of the interface model two different types of finite element discretizations were studied. Both discretizations are based on a discrete multiplier space defined on the fracture interface. However they differ in the choice of the mesh and the basis functions of the Lagrange multiplier.

The first of these relies on discontinuous piecewise constant basis functions on sufficiently large multiplier elements $\left(h_{\gamma} \geq 3 h\right)$. Under this condition, following [34], we proved existence, uniqueness and convergence of the discrete problem. Several numerical experiments confirmed the theoretical results and validated the approach. The numerical experiments showed that in practice the mesh size of the multiplier can be chosen finer than the theoretical results suggest. Provided that $h_{\lambda} \geq$ $\min \left\{h, h_{\gamma}\right\}$, a multiplier mesh size in the range of the matrix element, i.e. $h_{\lambda} \approx h$, yields a reasonable balance between accuracy, convergence and conditioning.

The second discretization is based on continuous, piecewise-affine, multiplier elements. In particular the mesh for the multiplier is the same as that for the fracture. In this case we could prove that there exists a unique solution of the discrete problem, but not convergence. However the numerical results indicated that the discrete problem converges to the reference solution and validated the approach. Similar to the previous discretization, mesh sizes of $h_{\lambda}=h_{\gamma} \approx h$ or $h_{\lambda}=h_{\gamma} \approx h / 2$ yield a reasonable balance between accuracy and convergence. We note though that the con- dition number is generally significantly higher for the second discretization for $\lambda_{h}\left(\mathbb{P}^{1}\right)$ than for the first $\left(\mathbb{P}^{0}\right)$.

The numerical examples showed that the errors of matrix and fracture pressure decrease linearly in the $H^{1}$ norm. However the errors deviate from the desired rates in the $L^{2}$ norm resulting from the nonconforming fashion the fracture intersects the matrix elements and the regularity of the different test cases. Therefore the $L^{2}$-convergence generally varies between $O(h)$ and $O\left(h^{2}\right)$. If the fracture mesh is aligned with the matrix mesh we obtain optimal rates of convergence. In accordance with the theoretical findings the Lagrange multiplier is characterized by rates up to $O\left(h_{\lambda}\right)$. The results of the benchmark problems in [27] lead us to the conclusion that the method is in good agreement with other fracture models and is an efficient alternative for the approximation of flow problems in fractured porous media.

Acknowledgements The authors would like to thank the German Research Foundation (DFG) for financial support of the project within the Cluster of Excellence in Simulation Technology (EXC 310/2) at the University of Stuttgart.

\section{References}

1. Ahmed, E., Jaffré, J., Roberts, J.E.: A reduced fracture model for two-phase flow with different rock types. Math. Comput. Simulation 137, 49-70 (2017)

2. Ahmed, R., Edwards, M.G., Lamine, S., Huisman, B.A.H., Pal, M.: Control-volume distributed multi-point flux approximation coupled with a lower-dimensional fracture model. J. Comput. Phys. 284, 462-489 (2015)

3. Ahmed, R., Edwards, M.G., Lamine, S., Huisman, B.A.H., Pal, M.: Three-dimensional control-volume distributed multi-point flux approximation coupled with a lower-dimensional surface fracture model. J. Comput. Phys. 303, 470-497 (2015)

4. Ainsworth, M.: A Posteriori Error Estimation for Discontinuous Galerkin Finite Element Approximation. SIAM J. Numer. Anal. 45(4), 1777-1798 (2007)

5. Alboin, C., Jaffré, J., Roberts, J.E.: Domain decomposition for flow in porous media with fractures. In: Domain Decomposition Methods in Sciences and Engineering. Domain Decomposition Press (1999)

6. Alboin, C., Jaffré, J., Roberts, J.E., Serres, C.: Modeling fractures as interfaces for flow and transport in porous media. In: Fluid flow and transport in porous media: mathematical and numerical treatment, Contemp. Math., vol. 295, pp. 13-24. Amer. Math. Soc., Providence, RI (2002)

7. Angot, P., Boyer, F., Hubert, F.: Numerical modelling of flow in fractured porous media. In: FVCA IV, pp. 249-260. ISTE, London (2005)

8. Antonietti, P.F., Facciolà, C., Russo, A., Verani, M.: Discontinuous Galerkin approximation of flows in fractured porous media on polytopic grids. Tech. Rep. 22/2016, Politecnico di Milano (2016)

9. Antonietti, P.F., Formaggia, L., Scotti, A., Verani, M., Verzott, N.: Mimetic finite difference approximation of flows in fractured porous media. ESAIM: M2AN 50(3), 809-832 (2016)

10. Baca, R.G., Arnett, R.C., Langford, D.W.: Modelling fluid flow in fractured-porous rock masses by finiteelement techniques. Int. J. Numer. Methods Fluids 4, 337-348 (1984) 
11. Berkowitz, B.: Characterizing flow and transport in fractured geological media: A review. Adv. Water Resour. 25(8-12), 861-884 (2002)

12. Berrone, S., Canuto, C., Pieraccini, S., Scialò, S.: Uncertainty quantification in Discrete Fracture Network models: Stochastic fracture transmissivity. Comput. Math. Appl. 70(4), 603-623 (2015)

13. Berrone, S., Pieraccini, S., Scialò, S.: An optimization approach for large scale simulations of discrete fracture network flows. J. Comput. Phys. 256, 838-853 (2014)

14. Boffi, D., Brezzi, F., Fortin, M.: Mixed finite element methods and applications, Springer Series in Computational Mathematics, vol. 44. Springer, Heidelberg (2013)

15. Boon, W., Nordbotten, J.: Robust Discretization of Flow in Fractured Porous Media. ArXiv e-print (2016). ArXiv:1601.06977 [math.NA]

16. Brenner, K., Groza, M., Guichard, C., Lebeau, G., Masson, R.: Gradient discretization of hybrid dimensional Darcy flows in fractured porous media. Numer. Math. 134(3), 569-609 (2016)

17. Brenner, K., Groza, M., Guichard, C., Masson, R.: Vertex approximate gradient scheme for hybrid dimensional twophase Darcy flows in fractured porous media. ESAIM Math. Model. Numer. Anal. 49(2), 303-330 (2015)

18. Brenner, K., Groza, M., Jeannin, L., Masson, R., Pellerin, J.: Immiscible two-phase Darcy flow model accounting for vanishing and discontinuous capillary pressures: application to the flow in fractured porous media. Comput. Geosci. (2017)

19. Brenner, S.C., Scott, L.R.: The Mathematical Theory of Finite Element Methods. Texts in Applied Mathematics. Springer New York (2002)

20. Brezzi, F.: On the existence, uniqueness and approximation of saddle-point problems arising from Lagrangian multipliers. RAIRO Anal. Numer. 2(10), 129-151 (1974)

21. Brezzi, F., Lipnikov, K., Simoncini, V.: A family of mimetic finite difference methods on polygonal and polyhedral meshes. Math. Models Methods Appl. Sci 15(10), 1533-1551 (2005)

22. Capatina, D., Luce, R., El-Otmany, H., Barrau, N.: Nitsche's extended finite element method for a fracture model in porous media. Appl. Anal. 95(10), 2224-2242 (2016)

23. D'Angelo, C., Scotti, A.: A mixed finite element method for Darcy flow in fractured porous media with nonmatching grids. ESAIM: Math. Model. Numer. Anal. 46(2), 465-489 (2012)

24. Ern, A., Guermond, J.L.: Theory and practice of finite elements, Applied Mathematical Sciences, vol. 159 Springer-Verlag, New York (2004)

25. Faille, I., Flauraud, E., Nataf, F., Pegaz-Fiornet, S., Schneider, F., Willien, F.: A new fault model in geological basin modelling, application to finite volume scheme and domain decomposition methods. In: FVCA III, pp. 543-550. Hermés Penton Sci. (2002)

26. Faille, I., Fumagalli, A., Jaffré, J., Roberts, J.E.: Model reduction and discretization using hybrid finite volumes for flow in porous media containing faults. Comput. Geosci. 20(2), 317-339 (2016)

27. Flemisch, B., Berre, I., Boon, W., Fumagalli, A., Schwenck, N., Scotti, A., Stefansson, I., Tatomir, A. Benchmarks for single-phase flow in fractured porous media. Adv. Water Resour. 111, 239-258 (2018)

28. Frih, N., Martin, V., Roberts, J.E., Saâda, A.: Modeling fractures as interfaces with nonmatching grids. Comput. Geosci. 16(4), 1043-1060 (2012)

29. Frih, N., Roberts, J.E., Saada, A.: Modeling fractures as interfaces: a model for Forchheimer fractures. Comput. Geosci. 12(1), 91-104 (2008)

30. Fumagalli, A., Scotti, A.: Numerical modelling of multiphase subsurface flow in the presence of fractures. Commun. Appl. Ind. Math. 3(1), e-380, 23 (2012)
31. Fumagalli, A., Scotti, A.: A numerical method for twophase flow in fractured porous media with non-matching grids. Adv. Water Resour. 62, Part C, 454-464 (2013). Computational Methods in Geologic CO2 Sequestration

32. Geiger, S., Dentz, M., Neuweiler, I.: A novel multi-rate dual-porosity model for improved simulation of fractured and multi-porosity reservoirs. Soc. Petrol. Eng. J. 18(4), 670-684 (2013)

33. Geiger, S., Matthai, S., Niessner, J., Helmig, R.: Blackoil simulations for three-component, three-phase flow in fractured porous media. Soc. Petrol. Eng. J. 14(2), 338$354(2009)$

34. Girault, V., Glowinski, R.: Error analysis of a fictitious domain method applied to a Dirichlet problem. Jpn. J. Ind. Appl. Math. 12(3), 487 (1995)

35. Gläser, D., Helmig, R., Flemisch, B., Class, H.: A discrete fracture model for two-phase flow in fractured porous media. Adv. Water Resour. 110, 335-348 (2017)

36. Hoteit, H., Firoozabadi, A.: An efficient numerical model for incompressible two-phase flow in fractured media. Adv. Water Resour. 31, 891-905 (2008)

37. Huang, H., Long, T.A., Wan, J., Brown, W.P.: On the use of enriched finite element method to model subsurface features in porous media flow problems. Comput. Geosci. 15(4), 721-736 (2011)

38. Jaffré, J., Mnejja, M., Roberts, J.E.: A discrete fracture model for two-phase flow with matrix-fracture interaction. Procedia Comput. Sci. 4, 967-973 (2011)

39. Karimi-Fard, M., Durlofsky, L.J., Aziz, K.: An efficient discrete-fracture model applicable for general-purpose reservoir simulators. Soc. Petrol. Eng. J. 9(2), 227-236 (2004)

40. Karimi-Fard, M., Firoozabadi, A.: Numerical simulation of water injection in 2D fractured media using discretefracture model. SPE Reservoir Eval. Eng. 4, 117-126 (2003)

41. Knabner, P., Roberts, J.E.: Mathematical analysis of a discrete fracture model coupling Darcy flow in the matrix with Darcy-Forchheimer flow in the fracture. ESAIM: Math. Model. Numer. Anal. 48(5), 1451-1472 (2014)

42. Köppel, M.: Flow in Heterogeneous Porous Media: Fractures and Uncertainty Quantification. Ph.D. thesis, University of Stuttgart, Germany (2018)

43. Köppel, M., Martin, V., Roberts, J.E.: A stabilized Lagrange multiplier finite-element method for flow in porous media with fractures. submitted (2018). URL https://hal.archives-ouvertes.fr/hal-01761591

44. Lesinigo, M., D'Angelo, C., Quarteroni, A.: A multiscale Darcy-Brinkman model for fluid flow in fractured porous media. Numer. Math. 117(4), 717-752 (2011)

45. List, F., Kumar, K., Pop, I.S., Radu, F.A.: Rigorous upscaling of unsaturated flow in fractured porous media. UHasselt Computational Mathematics Preprint (2018). Nr. UP-18-04

46. Martin, V., Jaffré, J., Roberts, J.E.: Modeling Fractures and Barriers as Interfaces for Flow in Porous Media. SIAM J. Sci. Comput. 26(5), 1667-1691 (2005)

47. Matthai, S.K., Mezentsev, A.A., Belayneh, M.: Finite Element?Node-Centered Finite-Volume Two-Phase-Flow Experiments With Fractured Rock Represented by Unstructured Hybrid-Element Meshes. SPE Reservoir Eval. Eng. 10 (2007)

48. Moinfar, A., Varavei, A., Sepehrnoori, K., Johns, R.T. Development of an Efficient Embedded Discrete Fracture Model for 3D Compositional Reservoir Simulation in Fractured Reservoirs. Soc. Petrol. Eng. J. (2014)

49. Monteagudo, J.E.P., Firoozabadi, A.: Control-volume method for numerical simulation of two-phase immiscible flow in two- and three-dimensional discrete-fractured media. Water Resour. Res. 40(7) (2004). W07405

50. Neuman, S.P.: Trends, prospects and challenges in quantifying flow and transport through fractured rocks. Hydrogeol. J. 13(1), 124-147 (2005) 
51. Pichot, G., Erhel, J., de Dreuzy, J.R.: A generalized mixed hybrid mortar method for solving flow in stochastic discrete fracture networks. SIAM J. Sci. Comput. 34(1), B86-B105 (2012)

52. Reichenberger, V., Jakobs, H., Bastian, P., Helmig, R.: A mixed-dimensional finite volume method for two-phase flow in fractured porous media. Adv. Water Resour. 29(7), 1020-1036 (2006)

53. Roberts, J.E., Thomas, J.M.: Mixed and hybrid methods. In: Handbook of numerical analysis, Vol. II, Handb. Numer. Anal., II, pp. 523-639. North-Holland, Amsterdam (1991)

54. Sandve, T.H., Berre, I., Nordbotten, J.M.: An efficient multi-point flux approximation method for Discrete Fracture-Matrix simulations. J. Comput. Phys. 231(9), 3784-3800 (2012)

55. Schwenck, N., Flemisch, B., Helmig, R., Wohlmuth, B.I. Dimensionally reduced flow models in fractured porous media: crossings and boundaries. Comput. Geosci. 19(6), 1219-1230 (2015)

56. Scott, L.R., Zhang, S.: Finite Element Interpolation of Nonsmooth Functions Satisfying Boundary Conditions. Math. Comput. 54(190), 483-493 (1990)

57. (SKI), S.N.P.I.: The international hydrocoin project background and results. OECD. Paris, France (1987)

58. Tunc, X., Faille, I., Gallouët, T., Cacas, M.C., Havé, P.: A model for conductive faults with non-matching grids. Comput. Geosci. 16(2), 277-296 (2011) 